\title{
Boundary correlators in WZW model on $\mathrm{AdS}_{2}$
}

\author{
Matteo Beccaria, ${ }^{a}$ Hongliang Jiang ${ }^{b}$ and Arkady A. Tseytlin ${ }^{c, 1}$ \\ ${ }^{a}$ Dipartimento di Matematica e Fisica Ennio De Giorgi, Università del Salento \& INFN, \\ Via Arnesano, Lecce 73100, Italy \\ ${ }^{b}$ Albert Einstein Center for Fundamental Physics, Institute for Theoretical Physics, \\ University of Bern, Sidlerstrasse 5, Bern 3012, Switzerland \\ ${ }^{c}$ Blackett Laboratory, Imperial College, \\ Prince Consort Road, London, U.K. \\ E-mail: matteo.beccaria@le.infn.it, jiang@itp.unibe.ch, \\ tseytlin@imperial.ac.uk
}

ABSTRACT: Boundary correlators of elementary fields in some $2 \mathrm{~d}$ conformal field theories defined on $\mathrm{AdS}_{2}$ have a particularly simple structure. For example, the correlators of the Liouville scalar happen to be the same as the correlators of the chiral component of the stress tensor on a plane restricted to the real line. Here we show that an analogous relation is true also in the WZW model: boundary correlators of the WZW scalars have the same structure as the correlators of chiral Kac-Moody currents. This is checked at the level of the tree and one-loop Witten diagrams in $\mathrm{AdS}_{2}$. We also compute some tree-level correlators in a generic $\sigma$-model defined on $\mathrm{AdS}_{2}$ and show that they simplify only in the WZW case where an extra Kac-Moody symmetry appears. In particular, the terms in 4point correlators having logarithmic dependence on 1d cross-ratio cancel only at the WZW point. One motivation behind this work is to learn how to compute $\mathrm{AdS}_{2}$ loop corrections in $2 \mathrm{~d}$ models with derivative interactions related to the study of correlators of operators on Wilson loops in string theory in AdS.

Keywords: AdS-CFT Correspondence, Conformal Field Models in String Theory ARXIV EPRINT: 2001.11269

\footnotetext{
${ }^{1}$ Also at the Institute for Theoretical and Mathematical Physics, Moscow State University and Lebedev Institute, Moscow.
} 


\section{Contents}

1 Introduction $\quad 2$

2 Boundary correlators in $\mathrm{SL}(2, \mathbb{R}) \mathrm{WZW}$ model on $\mathrm{AdS}_{2} \quad 4$

2.1 Action 5

2.2 Propagators 6

$\begin{array}{lll}2.3 & \text { Tree-level boundary correlation functions } & 6\end{array}$

$\begin{array}{lll}2.3 .1 & \text { Two- and three- point functions } & 6\end{array}$

$\begin{array}{lll}2.3 .2 & \text { Four-point functions } & 7\end{array}$

2.4 Matching $\mathrm{AdS}_{2}$ boundary correlators with correlators of chiral currents 10

$\begin{array}{lll}2.5 & \text { Boundary correlators in } \mathrm{PCM}_{q} \text { on } \mathrm{AdS}_{2} & 11\end{array}$

3 Tree-level boundary correlators in generic $\sigma$-model on $\mathbf{A d S}_{2} \quad 12$

$\begin{array}{lll}3.1 \text { Action } & 12\end{array}$

3.2 Tree-level $\mathrm{AdS}_{2}$ boundary correlation functions 13

$\begin{array}{ll}\text { 3.3 WZW model case: matching with correlators of chiral currents } & 16\end{array}$

4 Quantum corrections to boundary correlators in $\operatorname{SL}(2, \mathbb{R})$ WZW model 17

$\begin{array}{lll}4.1 & \text { One-loop corrections to the two-point correlators } & 18\end{array}$

$\begin{array}{lll}4.1 .1\langle\Psi \widetilde{\Psi}\rangle & 18\end{array}$

$\begin{array}{lll}4.1 .2\langle\Phi \Phi\rangle & 19\end{array}$

4.2 One-loop correction to the three-point correlator 21

5 Boundary correlators and scattering amplitudes on $\mathrm{AdS}_{2} \quad 23$

5.1 Massive scalar S-matrix on $\mathrm{AdS}_{2}$

5.1.1 Comments on relation to boundary correlators 25

5.1.2 Tree level scattering in Liouville theory on $\mathrm{AdS}_{2}$

$\begin{array}{ll}5.2 \text { Massless scattering case } & 27\end{array}$

$\begin{array}{llr}6 & \text { Concluding remarks } & 29\end{array}$

$\begin{array}{lr}\text { A Notation and conventions } & 29\end{array}$

B Global symmetry constraints in $\operatorname{SL}(2, \mathbb{R})$ WZW model 30

C Alternative computation of one-loop boundary correlators in $\operatorname{SL}(2, \mathbb{R})$

$\begin{array}{ll}\text { WZW model } & 31\end{array}$ 


\section{Introduction}

Study of $\sigma$-models in $\mathrm{AdS}_{2}$ is of interest for several reasons (see, e.g., [1-3]). Here we will consider correlators of elementary $\sigma$-model fields in Euclidean $\mathrm{AdS}_{2}$ with Poincare metric $d s^{2}=\frac{1}{z^{2}}\left(d \mathrm{t}^{2}+d \mathrm{z}^{2}\right)$. While in flat space the scattering amplitudes of massless scalar fields in perturbative vacuum are ambiguous due to IR divergences (see, e.g., [4]) the coordinate-space boundary correlators in $\mathrm{AdS}_{2}$ are well-defined and are constrained by $1 \mathrm{~d}$ conformal invariance. One interesting question is how the structure of these correlators is further restricted by hidden symmetries of the $\sigma$-model and how to compute $\mathrm{AdS}_{2}$ loop corrections in a way consistent with these underlying symmetries.

Since a classical $\sigma$-model in curved $2 \mathrm{~d}$ space is Weyl-invariant (with the scalar field not transforming), defined on $\mathrm{AdS}_{2}$ it is formally the same as on a half-plane $d s^{2}=d \mathrm{t}^{2}+d \mathrm{z}^{2}$, $z>0$. This is true also for models that are conformally invariant at quantum level, where Weyl anomaly is decoupled from $\sigma$-model correlators. Compared to a generic boundary CFT set-up here we are interested in (i) the standard $\mathrm{AdS}_{2}$ (or Dirichlet) boundary conditions $\left.\varphi(\mathrm{t}, \mathrm{z})\right|_{\mathrm{z} \rightarrow 0}=\mathrm{z}^{\Delta} \Phi(\mathrm{t})+\cdots$ for an elementary field with mass $m^{2}=\Delta(\Delta-1)$; (ii) correlators of elementary fields $\varphi$ rather than composite operators with good $2 \mathrm{~d}$ conformal transformation properties. The $1 \mathrm{~d}$ boundary operators dual to the massless $\sigma$-model fields with Dirichlet b.c. will thus have $\Delta=1$. In contrast to the Liouville theory case discussed in [5-7] it turns out that the classical $2 \mathrm{~d}$ conformal invariance of the bulk $\sigma$-model theory does not sufficiently constrain the structure of the tree-level boundary correlators. For example, the tree-level boundary four-point functions are still non-trivial log functions of $1 \mathrm{~d}$ cross-ratio. An important difference is that while the $\sigma$-model field is a scalar on which conformal symmetry acts trivially, the Liouville field transforms non-trivially under the conformal transformations. ${ }^{1}$ Similar tree-level correlators (containing logs) were found also for the fields of the Nambu action in $\mathrm{AdS}_{2}[1,2]$, but they appear already in the case of 2-derivative $\sigma$-model vertices.

To study the role of additional $\sigma$-model symmetries here we will consider the example of the WZW model $[13,14]$ which has an infinite-dimensional Kac-Moody (KM) symmetry $g^{\prime}=u(w) g v(\bar{w}), w=\mathrm{t}+i z$. It appears for the special value of the ratio of the coefficients of the principal chiral model (PCM) and WZ terms in the action when the classical equations of motion admit a chiral decomposition (the resulting model is then conformal and KM invariant also at the quantum level). Like the Virasoro symmetry in the Liouville case here the KM symmetry will impose rigid constraints on the $\mathrm{AdS}_{2}$ boundary correlators of the elementary fields $\varphi_{a}$ parametrizing $g$. In particular, the KM symmetry rules out the presence of $\log$ terms in the four-point correlators, both at the tree and the quantum level.

As we will argue below, the $\mathrm{AdS}_{2}$ boundary correlators of the massless fields $\varphi_{a}$ defined in the standard way as

$$
\left\langle\Phi_{a_{1}}\left(\mathrm{t}_{1}\right) \cdots \Phi_{a_{n}}\left(\mathrm{t}_{n}\right)\right\rangle \equiv \lim _{\mathrm{z}_{i} \rightarrow 0} \prod_{i=1}^{n} \mathrm{z}_{i}^{-\Delta}\left\langle\varphi_{a_{1}}\left(\mathrm{t}_{1}, \mathrm{z}_{1}\right) \cdots \varphi_{a_{n}}\left(\mathrm{t}_{n}, \mathrm{z}_{n}\right)\right\rangle_{\mathrm{AdS}_{2}}, \quad \Delta=1,
$$

\footnotetext{
${ }^{1}$ The theories in flat space and in $\mathrm{AdS}_{2}$ correspond to different vacua [5, 8, 9]. The Liouville field in $\mathrm{AdS}_{2}$ has a constant vacuum with the fluctuation field with $m^{2}=2$ and thus $\Delta=2$. Its boundary correlators are constrained by $1 \mathrm{~d}$ Virasoro symmetry and thus are exactly the same as the $2 \mathrm{~d}$ stress-tensor correlators restricted to the boundary $[6,7]$. This generalizes also to the Toda theory (see also [10-12]).
} 
are constrained by the underlying KM symmetry so that they are equal, up to a universal prefactor, to the correlators of the chiral component of the WZW current $J^{a}(w) \sim$ $\operatorname{tr}\left(t^{a} \partial_{w} g g^{-1}\right), w=\mathrm{t}+i z$, restricted to the boundary. This is formally equivalent to the "identification" of the boundary operator associated to $\varphi_{a}$ with the chiral component of the current $J_{a}(w \rightarrow \mathrm{t})$

$$
\left.\Phi_{a}(\mathrm{t}) \rightarrow \kappa J_{a}(w)\right|_{\mathrm{z} \rightarrow 0}, \quad \kappa=\sqrt{\frac{2}{k}} .
$$

Here $k$ is the WZW level. For comparison, in the Liouville theory case the role of the $\Delta=1$ current $J \equiv J_{w}$ (the generator of KM symmetry) is played by the $\Delta=2$ chiral stress tensor $T \equiv T_{w w}$ (the generator of the Virasoro symmetry) ${ }^{2}$ and the proportionality coefficient was $\kappa=-4 \sqrt{\frac{c-1}{6 c^{2}}}$ where $c=1+6\left(b^{-1}+b\right)^{2}$ is the Liouville central charge [7]. ${ }^{3}$ In the WZW case the KM symmetry implies the Virasoro symmetry but is much stronger: as already mentioned above, the boundary correlators in conformal $\sigma$-models that do not have an extra KM symmetry have much more complicated structure. ${ }^{4}$

Explicitly, the standard OPE relation for the chiral components of the KM current (see, e.g., [19])

$$
J^{a}(w) J^{b}\left(w^{\prime}\right) \sim \frac{k \delta^{a b}}{\left(w-w^{\prime}\right)^{2}}+\frac{f^{a b c} J^{c}(w)}{w-w^{\prime}}+\cdots,
$$

determines all higher current correlators to be given by

$$
\begin{aligned}
\left\langle J^{a_{1}}\left(w_{1}\right) J^{a_{2}}\left(w_{2}\right)\right\rangle & =\frac{k \delta^{a_{1} a_{2}}}{\left(w_{1}-w_{2}\right)^{2}}, \\
\left\langle J^{a_{1}}\left(w_{1}\right) J^{a_{2}}\left(w_{2}\right) J^{a_{3}}\left(w_{3}\right)\right\rangle & =\frac{k f^{a_{1} a_{2} a_{3}}}{w_{12} w_{13} w_{23}}, \\
\left\langle J^{a_{1}}\left(w_{1}\right) J^{a_{2}}\left(w_{2}\right) J^{a_{3}}\left(w_{3}\right) J^{a_{4}}\left(w_{4}\right)\right\rangle & =\frac{k^{2} \delta^{a_{1} a_{2}} \delta^{a_{3} a_{4}}}{w_{12}^{2} w_{34}^{2}}+\frac{k f^{a_{1} a_{2} b} f^{a_{3} a_{4} b}}{w_{12} w_{34} w_{23} w_{24}}+(2 \leftrightarrow 3)+(2 \leftrightarrow 4) .
\end{aligned}
$$

Below we will explicitly reproduce (1.4), (1.5), (1.6) with $w_{i} \rightarrow \mathrm{t}_{i}$ as the expressions for the boundary correlators of the WZW fields (1.1) computed in the $1 / k$ perturbation theory in $\mathrm{AdS}_{2}$ with the identification (1.2).

A semiclassical argument of why the boundary correlators of $\varphi_{a}$ are related to the restriction of the current correlators to the boundary of half-plane can be given as follows

\footnotetext{
${ }^{2}$ In the Liouville (or Toda) case the Virasoro symmetry becomes realized as a reparametrizations of the boundary and thus completely fixes the structure of the correlators modulo overall powers of the coordinateindependent factor $\kappa$.

${ }^{3}$ This close analogy may not be accidental given that the Liouville theory may be obtained by a Hamiltonian reduction from the SL(2) WZW model $[15,16]$.

${ }^{4}$ The key point is that the elementary $\sigma$-model field transforms non-trivially under the KM symmetry (like the Liouville field was transforming under the conformal symmetry). Note also that the simplification of the form of boundary correlators in the case of KM symmetry is analogous to what happens in the AdS/CFT examples when the bulk theory has higher symmetry thus constraining also the correlators of the dual boundary CFT. An example is provided by the vectorial AdS/CFT where the symmetry in question is a higher spin symmetry $[17,18]$.
} 
(for a similar though more involved argument in the Liouville theory case see [7]). Starting with the expression $J^{a} \sim \operatorname{tr}\left(t^{a} \partial_{w} g g^{-1}\right) \rightarrow \partial_{w} \varphi^{a}+\mathcal{O}\left(\varphi^{2}\right)$ (where $\left.\partial_{w}=\frac{1}{2}\left(\partial_{z}-i \partial_{\mathrm{t}}\right)\right)$ and using the boundary condition $\left.\varphi_{a}(\mathrm{z}, \mathrm{t})\right|_{\mathrm{z} \rightarrow 0} \rightarrow \mathrm{z} \Phi_{a}(\mathrm{t})+\ldots$ we find that (up to an overall normalization constant) $\left.J^{a}\right|_{z \rightarrow 0} \rightarrow \Phi^{a}$.

To demonstrate the correspondence (1.2) we shall start in section 2 with the example of the $\mathrm{SL}(2, \mathbb{R})$ WZW model on $\mathrm{AdS}_{2}$ and compute boundary correlators of its fields in the leading tree-level approximation. We shall also consider the corresponding $\mathrm{PCM}_{q}$ theory (i.e. the PCM with a WZ term with coefficient $\propto q$ ), and show that the four-point correlators simplify (with logs of coordinates cancelling out) and thus can be matched with the correlators of the chiral currents only at the WZW point $\left(q^{2}=1\right)$ when the model has an extra KM symmetry. In section 3 we shall repeat the computation of the tree boundary correlators for a generic $\sigma$-model including the case of $\mathrm{PCM}_{q}$ for an arbitrary group $G$.

In section 4 we shall test the relation between the boundary correlators of the WZW fields and the chiral currents (1.2) beyond the classical (large $k$ ) limit by computing the one-loop corrections to the two-point and three-point boundary correlators. Like in similar computations in the Liouville and Toda theories in $\operatorname{AdS}_{2}[7,11,12]$ this requires an explicit evaluation of loop integrals in $\mathrm{AdS}_{2}$ which is subtle in the present case of the $\sigma$-model theory with two derivatives in the vertices. We shall argue that there exists a particular computational scheme in which the one-loop terms in the WZW field boundary correlators vanish, implying that the proportionality coefficient $\kappa$ in (1.2) does not receive $1 / k$ correction and thus its expression in (1.2) is expected to be exact.

It is interesting to note that while in flat space the scattering amplitudes for the massless WZW fields vanish $[4,20]$ their coordinate-space boundary correlators in $\mathrm{AdS}_{2}$ are non-vanishing. Their structure, however, is simple being dictated by the KM symmetry. One may wonder if with some natural definition of the S-matrix in AdS they may actually correspond to trivial scattering in $\mathrm{AdS}_{2}$ or on half-plane. We will address this question in section 5. There is a close analogy with what happens in the Liouville theory [5] where the full quantum S-matrix was argued to be trivial [21, 22]. We shall discuss the idea of defining $\mathrm{AdS}_{2}$ scattering amplitudes by Fourier transform of boundary correlators or using the prescription of [5] (cf. [23]) and argue that this leads to trivial three-point scattering amplitudes also in the present WZW case.

Some concluding remarks will be made in section 6. Appendix A will list our notation and conventions. In appendix B we shall discuss a constraint imposed by global symmetry on boundary two-point functions in the $\mathrm{SL}(2, \mathbb{R})$ WZW model. In appendix $\mathrm{C}$ we shall revisit the computation of the one-loop corrections to the two-point boundary correlators in $\mathrm{SL}(2, \mathbb{R})$ WZW model using an alternative form of the action and emphasizing some subtle scheme-dependence issues.

\section{Boundary correlators in $\mathrm{SL}(2, \mathbb{R})$ WZW model on $\mathrm{AdS}_{2}$}

To demonstrate the correspondence (1.2) we shall first consider the example of the $\mathrm{SL}(2, \mathbb{R})$ WZW model and compute its boundary correlators on $\mathrm{AdS}_{2}$ in the leading-order (tree) approximation. It is useful to view this WZW model as a special case of the $\mathrm{PCM}_{q}$, i.e. 
the principal chiral model with an additional WZ term. This allows one to investigate the consequences of the Kac-Moody symmetry appearing at the WZW point for the structure of the boundary correlators.

\subsection{Action}

The action for the $\mathrm{PCM}_{q}$ may be written as

$$
S=\frac{1}{2 \pi \lambda^{2}}\left[-\frac{1}{2} \int_{\Sigma} d^{2} x \operatorname{Tr}\left(g^{-1} \partial_{\mu} g g^{-1} \partial^{\mu} g\right)+\frac{i}{3} q \int_{\mathbf{B}^{3}} \operatorname{Tr}\left(g^{-1} d g \wedge g^{-1} d g \wedge g^{-1} d g\right)\right], q=\frac{1}{2} k \lambda^{2},
$$

where $k$ is the coefficient of the WZ term, $\Sigma$ is a Riemann surface and $\mathbf{B}^{3}$ is the $3 \mathrm{~d}$ extension of $\Sigma$ such that $\partial \mathbf{B}^{3}=\Sigma$. When

$$
\lambda=\sqrt{\frac{2}{|k|}}, \quad \text { i.e. } \quad q=\operatorname{sign} k= \pm 1,
$$

the action (2.1) reduces to the WZW model action.

Assuming $k>0$, a generic $\operatorname{SL}(2, \mathbb{R})$ group element may be represented in the Gauss decomposition form (see, e.g., [24])

$$
g(x)=\left(\begin{array}{ll}
1 & \psi \\
0 & 1
\end{array}\right)\left(\begin{array}{cc}
e^{-\frac{\lambda}{\sqrt{2}} \phi} & 0 \\
0 & e^{\frac{\lambda}{\sqrt{2}} \phi}
\end{array}\right)\left(\begin{array}{cc}
1 & 0 \\
\frac{\lambda^{2}}{2} \tilde{\psi} & 1
\end{array}\right) .
$$

Then the action (2.1) (written on generic curved 2-space with metric g) becomes

$$
S=\frac{1}{4 \pi} \int d^{2} x \sqrt{\mathrm{g}}\left[\mathrm{g}^{\mu \nu} \partial_{\mu} \phi \partial_{\nu} \phi+e^{b \phi}\left(\mathrm{g}^{\mu \nu}+i q \epsilon^{\mu \nu}\right) \partial_{\mu} \psi \partial_{\nu} \tilde{\psi}\right], \quad b \equiv \sqrt{2} \lambda .
$$

Here $\epsilon^{\mu \nu}=\frac{\varepsilon^{\mu \nu}}{\sqrt{\mathrm{g}}}$ is the standard antisymmetric tensor. This action may be interpreted as that of a $\sigma$-model with $\mathrm{AdS}_{3}$ target space and particular $B$-field coupling.

Specializing to the $q=1 \mathrm{WZW}$ point and the Euclidean $\mathrm{AdS}_{2}$ background (see appendix A for our notation and conventions)

$$
d s^{2}=\frac{d \mathrm{t}^{2}+d \mathrm{z}^{2}}{\mathrm{z}^{2}}=-4 \frac{d w d \bar{w}}{(w-\bar{w})^{2}}, \quad w=\mathrm{t}+i \mathrm{z}, \quad \bar{w}=\mathrm{t}-i \mathrm{z}, \quad \mathrm{z}>0,
$$

we get the following expression for the corresponding $\mathrm{SL}(2, \mathbb{R})$ WZW action

$$
S=\int \mathrm{d}^{2} w\left(\partial \phi \bar{\partial} \phi+e^{b \phi} \partial \psi \bar{\partial} \tilde{\psi}\right)=\int \mathrm{d}^{2} w\left(\partial \phi \bar{\partial} \phi+\partial \psi \bar{\partial} \tilde{\psi}+b \phi \partial \psi \bar{\partial} \tilde{\psi}+\frac{1}{4} b^{2} \phi^{2} \partial \psi \bar{\partial} \tilde{\psi}+\cdots\right), \quad b=\frac{2}{\sqrt{k}} .
$$

As the conformal factor of the metric decouples, this is formally the same as the WZW action on a flat half-plane $z>0$. However, it will be useful to phrase the computation of the boundary correlators in the $\mathrm{AdS}_{2}$ language.

We shall assume that the massless fields $\phi, \psi, \tilde{\psi}$ are subject to the standard (Dirichlet) boundary conditions and thus they should be dual to the boundary operators with dimension $\Delta=1$, i.e. the asymptotic expansion of these fields near $\mathbf{z}=0$ is

$$
\mathrm{z} \rightarrow 0: \quad \phi(\mathrm{t}, \mathrm{z})=\mathrm{z} \Phi(\mathrm{t})+\cdots, \quad \psi(\mathrm{t}, \mathrm{z})=\mathrm{z} \Psi(\mathrm{t})+\cdots, \quad \tilde{\psi}(\mathrm{t}, \mathrm{z})=\mathrm{z} \widetilde{\Psi}(\mathrm{t})+\cdots
$$

Our aim will be to compute the tree level boundary correlation functions (1.1) for the fields in (2.6) and then match them with the correlators of KM currents. 


\subsection{Propagators}

The bulk-to-bulk propagator of a massless scalar in $\mathrm{AdS}_{2}$ with a standard normalization $\frac{1}{2} \int d^{2} w \partial^{\mu} \phi \partial_{\mu} \phi$ is

$$
G_{\Delta=1}(\eta)=-\frac{1}{4 \pi} \log \eta,
$$

where the geodesic distance is defined as

$$
\eta=\frac{u}{u+2}, \quad u=\frac{\left(\mathrm{t}-\mathrm{t}^{\prime}\right)^{2}+\left(\mathrm{z}-\mathrm{z}^{\prime}\right)^{2}}{2 \mathrm{zz}^{\prime}} .
$$

Hence, for the field $\phi$ in (2.6) we have (cf. $(\text { A.7) })^{5}$

$$
g_{\phi \phi}\left(w, w^{\prime}\right)=\left\langle\phi(w) \phi\left(w^{\prime}\right)\right\rangle=\stackrel{w \quad w^{\prime}}{\longrightarrow}=2 \pi G_{\Delta=1}(\eta) \equiv g(\eta)=-\frac{1}{2} \log \eta\left(w, w^{\prime}\right) .
$$

The bulk-to-bulk propagator of the pair of fields $\psi, \tilde{\psi}$ is similarly

$$
g_{\psi \tilde{\psi}}\left(w, w^{\prime}\right)=\stackrel{w \longrightarrow w^{\prime}}{\longrightarrow}=\left\langle\psi(w) \tilde{\psi}\left(w^{\prime}\right)\right\rangle=2 g\left(w, w^{\prime}\right)=-\log \eta\left(w, w^{\prime}\right) .
$$

Given the structure of the perturbative (small $b$ or large $k$ ) expansion in (2.6), it is useful also to quote the propagators for the differentiated fields

$$
\begin{aligned}
g_{\partial \psi \bar{\partial} \tilde{\psi}}\left(w, w^{\prime}\right) & =\left\langle\partial \psi(w) \bar{\partial} \tilde{\psi}\left(w^{\prime}\right)\right\rangle=\stackrel{\partial \psi(w)}{\longrightarrow} \bar{\partial} \tilde{\psi}\left(w^{\prime}\right) \\
& =\partial_{w} \bar{\partial}_{w^{\prime}} g_{\psi \tilde{\psi}}\left(w, w^{\prime}\right)=\partial_{w}\left(\frac{1}{\bar{w}-\bar{w}^{\prime}}-\frac{1}{w-\bar{w}^{\prime}}\right)=\frac{1}{\left(w-\bar{w}^{\prime}\right)^{2}}+\pi \delta^{(2)}\left(w-w^{\prime}\right),
\end{aligned}
$$

where we used the relations (A.6). The $\delta$-function piece here will be important to account for below.

To compute the boundary correlators, we will also need the bulk-to-boundary propagators

$$
\begin{gathered}
g_{\phi \phi}^{\partial}\left(\mathrm{t} ; w^{\prime}\right)=\lim _{\mathrm{z} \rightarrow 0} \frac{1}{\mathrm{z}} g_{\phi \phi}\left(\mathrm{t}, \mathrm{z} ; \mathrm{t}^{\prime}, \mathrm{z}^{\prime}\right)=\frac{2 \mathrm{z}^{\prime}}{\left(\mathrm{t}^{\prime}-\mathrm{t}\right)^{2}+\mathrm{z}^{\prime 2}}=\frac{-i}{\mathrm{t}-w^{\prime}}+\frac{i}{\mathrm{t}-\bar{w}^{\prime}} \equiv g^{\partial}\left(\mathrm{t} ; w^{\prime}\right), \\
g_{\psi \bar{\partial} \tilde{\psi}}^{\partial}\left(\mathrm{t} ; w^{\prime}\right)=\lim _{\mathrm{z} \rightarrow 0} \frac{1}{\mathrm{z}}\left\langle\psi(w) \bar{\partial} \tilde{\psi}\left(w^{\prime}\right)\right\rangle=\frac{2 i}{\left(\mathrm{t}-\bar{w}^{\prime}\right)^{2}}=\bar{\partial}_{w^{\prime}} \frac{2 i}{\left(\mathrm{t}-\bar{w}^{\prime}\right)}=2 \bar{\partial}^{\prime} g^{\partial}\left(\mathrm{t} ; w^{\prime}\right), \\
g_{\tilde{\psi} \partial \psi}^{\partial}\left(\mathrm{t} ; w^{\prime}\right)=\lim _{\mathrm{z} \rightarrow 0} \frac{1}{\mathrm{z}}\left\langle\tilde{\psi}(w) \partial \psi\left(w^{\prime}\right)\right\rangle=\frac{-2 i}{\left(\mathrm{t}-w^{\prime}\right)^{2}}=\partial_{w^{\prime}} \frac{-2 i}{\left(\mathrm{t}-w^{\prime}\right)}=2 \partial^{\prime} g^{\partial}\left(\mathrm{t} ; w^{\prime}\right) .
\end{gathered}
$$

\subsection{Tree-level boundary correlation functions}

\subsubsection{Two- and three- point functions}

Considering the boundary-to-boundary case of the propagators (2.10), we get the following two-point functions (using the notation in (1.1))

$$
\left\langle\Phi\left(\mathrm{t}_{1}\right) \Phi\left(\mathrm{t}_{2}\right)\right\rangle=\frac{2}{\mathrm{t}_{12}^{2}}, \quad\left\langle\Psi\left(\mathrm{t}_{1}\right) \widetilde{\Psi}\left(\mathrm{t}_{2}\right)\right\rangle=\frac{4}{\mathrm{t}_{12}^{2}}, \quad \quad \mathrm{t}_{i j} \equiv \mathrm{t}_{i}-\mathrm{t}_{j} .
$$

These have the same form as the boundary restriction of (1.4).

\footnotetext{
${ }^{5}$ Note that $w, w^{\prime}$ in the propagators are labels of the points on half-plane: the propagators may also depend on the anti-holomorphic coordinates $\bar{w}, \bar{w}^{\prime}$ but we do not indicate this to simplify the notation.
} 
The only non-zero three-point function is $\langle\Phi \psi \widetilde{\Psi}\rangle$, which, at the tree level (leading order in $1 / k)$, is computed by the Witten diagram ${ }^{6}$

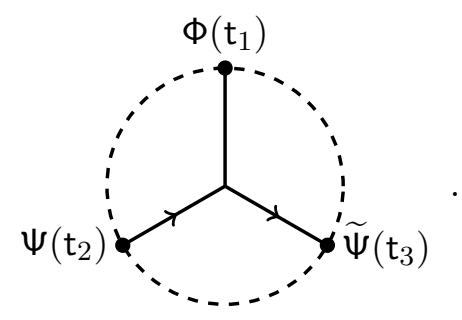

We have

$$
A_{3}\left(\mathrm{t}_{1}, \mathrm{t}_{2}, \mathrm{t}_{3}\right) \equiv\left\langle\Phi\left(\mathrm{t}_{1}\right) \Psi\left(\mathrm{t}_{2}\right) \widetilde{\Psi}\left(\mathrm{t}_{3}\right)\right\rangle=-b \int \mathrm{d}^{2} w g_{\phi \phi}^{\partial}\left(\mathrm{t}_{1}, w\right) g_{\psi \bar{\partial} \tilde{\psi}}^{\partial}\left(\mathrm{t}_{2}, w\right) g_{\tilde{\psi} \partial \psi}^{\partial}\left(\mathrm{t}_{3}, w\right) .
$$

Using the propagators in (2.13), we get

$$
A_{3}\left(\mathrm{t}_{1}, \mathrm{t}_{2}, \mathrm{t}_{3}\right)=-8 b \int_{0}^{\infty} d \mathrm{z} \int_{-\infty}^{\infty} d \mathrm{t} \frac{\mathrm{z}}{\pi\left[\left(\mathrm{t}-\mathrm{t}_{1}\right)^{2}+\mathrm{z}^{2}\right]\left(-\mathrm{t}+\mathrm{t}_{2}+i \mathrm{z}\right)^{2}\left(\mathrm{t}-\mathrm{t}_{3}+i \mathrm{z}\right)^{2}}
$$

This integral can be done by first computing the residues in the $t$ integration variable. Integrating then over $z$ one finds

$$
\left\langle\Phi\left(\mathrm{t}_{1}\right) \Psi\left(\mathrm{t}_{2}\right) \widetilde{\Psi}\left(\mathrm{t}_{3}\right)\right\rangle=\frac{4 i b}{\mathrm{t}_{12} \mathrm{t}_{23} \mathrm{t}_{31}} .
$$

This has again the same structure as the real-line limit of (1.5).

\subsubsection{Four-point functions}

We now turn to the four-point functions the computation of which is little more involved. The only non-vanishing cases are the correlators $\left\langle\Psi^{2} \widetilde{\Psi}^{2}\right\rangle$ and $\left\langle\Phi^{2} \Psi \widetilde{\Psi}\right\rangle$.

$\left\langle\boldsymbol{\Psi}^{2} \widetilde{\boldsymbol{\Psi}}^{2}\right\rangle$. At tree level this correlator is given by the following Witten diagrams

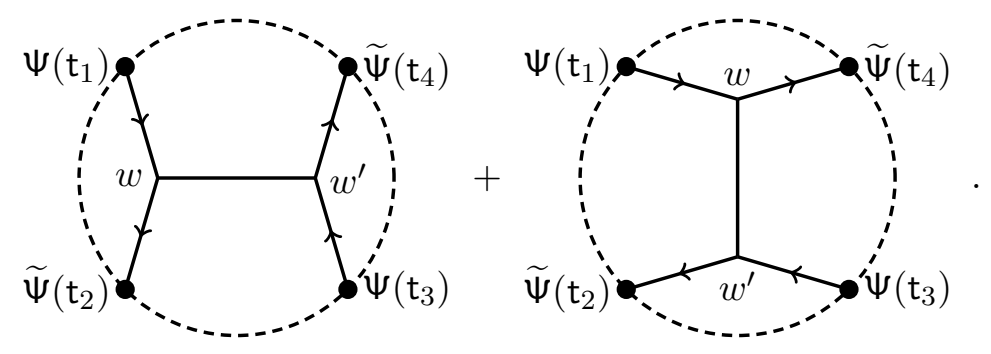

We can represent the result as

$$
\left\langle\Psi\left(\mathrm{t}_{1}\right) \widetilde{\Psi}\left(\mathrm{t}_{2}\right) \Psi\left(\mathrm{t}_{3}\right) \widetilde{\Psi}\left(\mathrm{t}_{4}\right)\right\rangle=A_{4}\left(\mathrm{t}_{1}, \mathrm{t}_{2}, \mathrm{t}_{3}, \mathrm{t}_{4}\right)+A_{4}\left(\mathrm{t}_{1}, \mathrm{t}_{4}, \mathrm{t}_{3}, \mathrm{t}_{2}\right),
$$

\footnotetext{
${ }^{6}$ Here the dashed circle represents the boundary of $\mathrm{AdS}_{2}$ and solid lines represent the propagators of the corresponding fields in the bulk.
} 
where

$$
\begin{aligned}
A_{4}\left(\mathrm{t}_{1}, \mathrm{t}_{2}, \mathrm{t}_{3}, \mathrm{t}_{4}\right) & =(-b)^{2} \int \mathrm{d}^{2} w \mathrm{~d}^{2} w^{\prime} g_{\psi \bar{\partial} \tilde{\psi}}^{\partial}\left(\mathrm{t}_{1}, w\right) g_{\tilde{\psi} \partial \psi}^{\partial}\left(\mathrm{t}_{2} ; w\right) g_{\phi \phi}\left(w, w^{\prime}\right) g_{\psi \bar{\partial} \tilde{\psi}}^{\partial}\left(\mathrm{t}_{3}, w^{\prime}\right) g_{\tilde{\psi} \partial \psi}^{\partial}\left(\mathrm{t}_{4} ; w^{\prime}\right) \\
& =2^{4} b^{2} \widetilde{\mathrm{H}}\left(\mathrm{t}_{2}, \mathrm{t}_{1}, \mathrm{t}_{4}, \mathrm{t}_{3}\right), \\
\widetilde{\mathrm{H}}\left(\mathrm{t}_{1}, \mathrm{t}_{2}, \mathrm{t}_{3}, \mathrm{t}_{4}\right) & \equiv \int \mathrm{d}^{2} w \mathrm{~d}^{2} w^{\prime} \partial g^{\partial}\left(\mathrm{t}_{1}, w\right) \bar{\partial} g^{\partial}\left(\mathrm{t}_{2} ; w\right) g\left(w, w^{\prime}\right) \partial^{\prime} g^{\partial}\left(\mathrm{t}_{3}, w^{\prime}\right) \bar{\partial}^{\prime} g^{\partial}\left(\mathrm{t}_{4} ; w^{\prime}\right) .
\end{aligned}
$$

To compute this integral we may first integrate by parts at the vertex $w$,

$$
\begin{aligned}
\widetilde{\mathbf{H}}\left(\mathrm{t}_{1}, \mathrm{t}_{2}, \mathrm{t}_{3}, \mathrm{t}_{4}\right) & =-\int \mathrm{d}^{2} w \mathrm{~d}^{2} w^{\prime} \frac{-i}{\mathrm{t}_{1}-w} \bar{\partial} g^{\partial}\left(\mathrm{t}_{2} ; w\right) \partial g\left(w, w^{\prime}\right) \partial^{\prime} g^{\partial}\left(\mathrm{t}_{3}, w^{\prime}\right) \bar{\partial}^{\prime} g^{\partial}\left(\mathrm{t}_{4} ; w^{\prime}\right) \quad(2.23) \\
& =\int d^{2} w d^{2} w^{\prime} \frac{-i \mathbf{z}^{\prime}}{\pi^{2}\left(-\bar{w}+\mathrm{t}_{2}\right)^{2}\left(w-\mathrm{t}_{1}\right)\left(\mathrm{t}_{4}-\bar{w}^{\prime}\right)^{2}\left(-\mathrm{t}_{3}+w^{\prime}\right)^{2}\left(w-w^{\prime}\right)\left(w-\bar{w}^{\prime}\right)},
\end{aligned}
$$

where we ignored 2-derivative terms assuming

$$
\partial^{\prime} \bar{\partial}^{\prime} g^{\partial}\left(\mathrm{t} ; w^{\prime}\right)=0 .
$$

Indeed, possible terms with $\delta^{(2)}\left(\mathrm{t}-w^{\prime}\right)$ and its derivative may be neglected here as they localize the bulk point to the boundary, and hence give zero contributions after performing the bulk integral.

The integral in (2.23) can be evaluated by applying the residue theorem

$$
\widetilde{\mathrm{H}}\left(\mathrm{t}_{1}, \mathrm{t}_{2}, \mathrm{t}_{3}, \mathrm{t}_{4}\right)=\frac{\log \left(\frac{\mathrm{t}_{12} \mathrm{t}_{34}}{\mathrm{t}_{14} \mathrm{t}_{23}}\right)^{2}+i \pi\left(\operatorname{sgn} \mathrm{t}_{12}+\operatorname{sgn} \mathrm{t}_{23}+\operatorname{sgn} \mathrm{t}_{34}+\operatorname{sgn} \mathrm{t}_{41}\right)}{4 \mathrm{t}_{13}^{2} \mathrm{t}_{24}^{2}}-\frac{1}{2 \mathrm{t}_{12} \mathrm{t}_{13} \mathrm{t}_{24} \mathrm{t}_{34}} .
$$

Then from (2.20) we finally obtain

$$
\left\langle\Psi\left(\mathrm{t}_{1}\right) \widetilde{\Psi}\left(\mathrm{t}_{2}\right) \Psi\left(\mathrm{t}_{3}\right) \widetilde{\Psi}\left(\mathrm{t}_{4}\right)\right\rangle=2^{4} b^{2} \widetilde{\mathrm{H}}\left(\mathrm{t}_{2}, \mathrm{t}_{1}, \mathrm{t}_{4}, \mathrm{t}_{3}\right)+2^{4} b^{2} \widetilde{\mathrm{H}}\left(\mathrm{t}_{4}, \mathrm{t}_{1}, \mathrm{t}_{2}, \mathrm{t}_{3}\right)=\frac{8 b^{2}}{\mathrm{t}_{12} \mathrm{t}_{23} \mathrm{t}_{34} \mathrm{t}_{41}} .
$$

Remarkably, all logarithmic (and sign function) terms present in (2.25) cancel out in the sum of the two exchange Witten diagrams. This cancellation is crucial in order to be able to match (2.26) with the correlators of the KM currents that are rational functions of the differences of points (cf. (1.6)).

$\left\langle\boldsymbol{\Phi}^{2} \boldsymbol{\Psi} \widetilde{\boldsymbol{\Psi}}\right\rangle$. This correlator is given by the sum of the following three diagrams
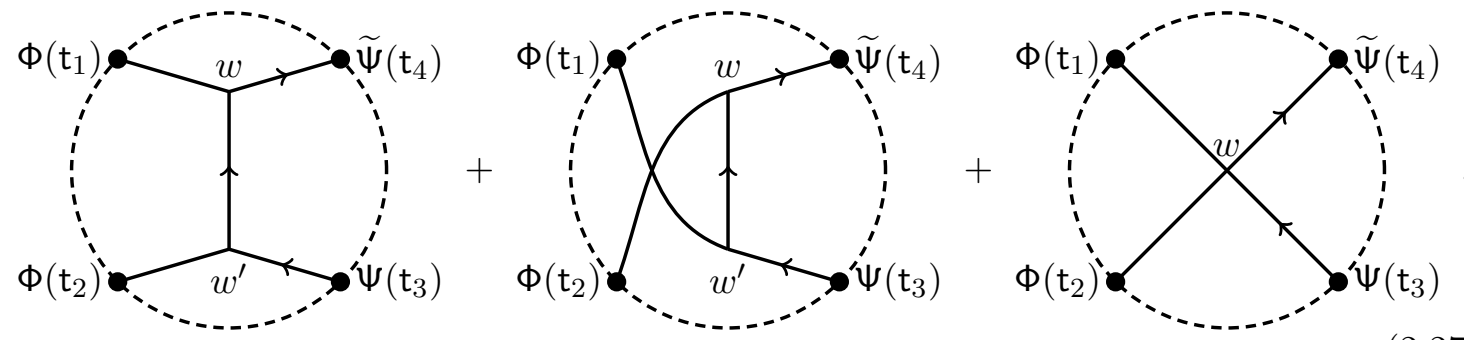

It can be written as

$$
\left\langle\Phi\left(\mathrm{t}_{1}\right) \Phi\left(\mathrm{t}_{2}\right) \Psi\left(\mathrm{t}_{3}\right) \widetilde{\Psi}\left(\mathrm{t}_{4}\right)\right\rangle=B_{4}\left(\mathrm{t}_{1}, \mathrm{t}_{2}, \mathrm{t}_{3}, \mathrm{t}_{4}\right)+B_{4}\left(\mathrm{t}_{2}, \mathrm{t}_{1}, \mathrm{t}_{3}, \mathrm{t}_{4}\right)+C_{4}\left(\mathrm{t}_{1}, \mathrm{t}_{2}, \mathrm{t}_{3}, \mathrm{t}_{4}\right),
$$


where the explicit form of $B_{4}$ and $C_{4}$ is

$$
\begin{aligned}
B_{4}\left(\mathrm{t}_{1}, \mathrm{t}_{2}, \mathrm{t}_{3}, \mathrm{t}_{4}\right) & =b^{2} \int \mathrm{d}^{2} w \mathrm{~d}^{2} w^{\prime} g_{\phi \phi}^{\partial}\left(\mathrm{t}_{1}, w\right) g_{\phi \phi}^{\partial}\left(\mathrm{t}_{2} ; w^{\prime}\right) g_{\partial \psi \bar{\partial} \tilde{\psi}}\left(w^{\prime}, w\right) g_{\psi \bar{\partial} \tilde{\psi}}^{\partial}\left(\mathrm{t}_{3}, w^{\prime}\right) g_{\tilde{\psi} \partial \psi}^{\partial}\left(\mathrm{t}_{4} ; w\right) \\
& =B_{4}^{\mathrm{reg}}\left(\mathrm{t}_{1}, \mathrm{t}_{2}, \mathrm{t}_{3}, \mathrm{t}_{4}\right)-C_{4}\left(\mathrm{t}_{1}, \mathrm{t}_{2}, \mathrm{t}_{3}, \mathrm{t}_{4}\right), \\
C_{4}\left(\mathrm{t}_{1}, \mathrm{t}_{2}, \mathrm{t}_{3}, \mathrm{t}_{4}\right) & =-b^{2} \int \mathrm{d}^{2} w g_{\phi \phi}^{\partial}\left(\mathrm{t}_{1}, w\right) g_{\phi \phi}^{\partial}\left(\mathrm{t}_{2} ; w\right) g_{\psi \bar{\partial} \tilde{\psi}}^{\partial}\left(\mathrm{t}_{3}, w\right) g_{\tilde{\psi} \partial \psi}^{\partial}\left(\mathrm{t}_{4} ; w\right) .
\end{aligned}
$$

Here $B_{4}^{\text {reg }}$ and $C_{4}$ are the contributions from the regular and singular parts of the internal propagators in (2.12), respectively (note that in (2.29) the singular $\delta$-function part in the propagator (2.12) turns the exchange diagram into a contact diagram). Using the explicit form of the propagators we get

$$
\begin{aligned}
B_{4}^{\mathrm{reg}}\left(\mathrm{t}_{1}, \mathrm{t}_{2}, \mathrm{t}_{3}, \mathrm{t}_{4}\right) & =\frac{16 b^{2}}{\pi^{2}} \int d^{2} w d^{2} w^{\prime} \frac{\mathrm{zz}^{\prime}}{\left(\mathrm{t}_{4}-\mathrm{t}-i \mathrm{z}\right)^{2}\left(\mathrm{t}_{3}-\mathrm{t}^{\prime}+i \mathrm{z}^{\prime}\right)^{2}\left[\left(\mathrm{t}-\mathrm{t}_{1}\right)^{2}+\mathrm{z}^{2}\right]\left[\left(\mathrm{t}^{\prime}-\mathrm{t}_{2}\right)^{2}+\mathrm{z}^{\prime 2}\right]} \\
& \times\left[-\mathrm{t}+\mathrm{t}^{\prime}+i\left(\mathrm{z}+\mathrm{z}^{\prime}\right)\right]^{-2} \\
C_{4}\left(\mathrm{t}_{1}, \mathrm{t}_{2}, \mathrm{t}_{3}, \mathrm{t}_{4}\right) & =\frac{-16 b^{2}}{\pi} \int d^{2} w \frac{\mathrm{z}^{2}}{\left(\mathrm{t}_{3}-\mathrm{t}+i \mathrm{z}\right)^{2}\left(\mathrm{t}_{4}-\mathrm{t}-i \mathrm{z}\right)^{2}\left[\left(\mathrm{t}_{1}-\mathrm{t}\right)^{2}+\mathrm{z}^{2}\right]\left[\left(\mathrm{t}_{2}-\mathrm{t}\right)^{2}+\mathrm{z}^{2}\right]} .
\end{aligned}
$$

Using the same method as for the previous four-point function, we obtain

$$
\begin{aligned}
& B_{4}^{\mathrm{reg}}\left(\mathrm{t}_{1}, \mathrm{t}_{2}, \mathrm{t}_{3}, \mathrm{t}_{4}\right)=-\frac{2 i b^{2}}{\mathrm{t}_{23}^{2} \mathrm{t}_{14}^{2}}\left[i \log \left(\frac{\mathrm{t}_{12} \mathrm{t}_{34}}{\mathrm{t}_{13} \mathrm{t}_{24}}\right)^{2}+\pi\left(-\operatorname{sgn} \mathrm{t}_{12}+\operatorname{sgn} \mathrm{t}_{13}-\operatorname{sgn} \mathrm{t}_{24}+\operatorname{sgn} \mathrm{t}_{34}\right)\right], \\
& C_{4}\left(\mathrm{t}_{1}, \mathrm{t}_{2}, \mathrm{t}_{3}, \mathrm{t}_{4}\right)=2 b^{2}\left[\frac{i \log \left(\frac{\mathrm{t}_{12} \mathrm{t}_{34}}{\mathrm{t}_{14} \mathrm{t}_{23}}\right)^{2}+\pi \operatorname{sgn}\left(\mathrm{t}_{12}\right)-\pi \operatorname{sgn}\left(\mathrm{t}_{14}\right)+\pi \operatorname{sgn}\left(\mathrm{t}_{23}\right)+\pi \operatorname{sgn}\left(\mathrm{t}_{34}\right)}{\mathrm{t}_{13}^{2} \mathrm{t}_{24}^{2}}\right. \\
&+i \frac{-i \log \left(\frac{\mathrm{t}_{12} \mathrm{t}_{34}}{\mathrm{t}_{13} \mathrm{t}_{24}}\right)^{2}+\pi \operatorname{sgn}\left(\mathrm{t}_{12}\right)-\pi \operatorname{sgn}\left(\mathrm{t}_{13}\right)+\pi \operatorname{sgn}\left(\mathrm{t}_{24}\right)-\pi \operatorname{sgn}\left(\mathrm{t}_{34}\right)}{\mathrm{t}_{14}^{2} \mathrm{t}_{23}^{2}} \\
&\left.+\frac{2}{\mathrm{t}_{13} \mathrm{t}_{14} \mathrm{t}_{23} \mathrm{t}_{24}}\right] .
\end{aligned}
$$

Inserting these results into $(2.28)$ gives $^{7}$

$$
\left\langle\Phi\left(\mathrm{t}_{1}\right) \Phi\left(\mathrm{t}_{2}\right) \Psi\left(\mathrm{t}_{3}\right) \widetilde{\Psi}\left(\mathrm{t}_{4}\right)\right\rangle=B_{4}^{\mathrm{reg}}\left(\mathrm{t}_{1}, \mathrm{t}_{2}, \mathrm{t}_{3}, \mathrm{t}_{4}\right)+B_{4}^{\mathrm{reg}}\left(\mathrm{t}_{2}, \mathrm{t}_{1}, \mathrm{t}_{3}, \mathrm{t}_{4}\right)-C_{4}\left(\mathrm{t}_{1}, \mathrm{t}_{2}, \mathrm{t}_{3}, \mathrm{t}_{4}\right)=-\frac{4 b^{2}}{\mathrm{t}_{13} \mathrm{t}_{14} \mathrm{t}_{23} \mathrm{t}_{24}} .
$$

\footnotetext{
${ }^{7}$ It is easy to verify that $B_{4}$ is related to $A_{4}$ in $(2.20)$ as $B_{4}\left(\mathrm{t}_{1}, \mathrm{t}_{2}, \mathrm{t}_{3}, \mathrm{t}_{4}\right)=A_{4}\left(\mathrm{t}_{1}, \mathrm{t}_{4}, \mathrm{t}_{3}, \mathrm{t}_{2}\right)$. This relation can be easily understood using integration by parts. Indeed, integrating by parts the cubic vertex, one can transfer the derivatives acting on the internal leg to the external legs; then the first diagram in (2.27) reduces to the second diagram in (2.19).
} 
As in the case of the correlator $\left\langle\Psi\left(\mathrm{t}_{1}\right) \widetilde{\Psi}\left(\mathrm{t}_{2}\right) \Psi\left(\mathrm{t}_{3}\right) \widetilde{\Psi}\left(\mathrm{t}_{4}\right)\right\rangle$ in $(2.26)$, both the logarithms and the sign functions again cancel out.

\subsection{Matching $\mathrm{AdS}_{2}$ boundary correlators with correlators of chiral currents}

Let us now compare the above boundary correlators with the correlators of the chiral WZW currents on the plane restricted to the real line. The correlation functions of the currents are a direct consequence of the KM algebra (1.3). Adapted to the $\mathrm{SL}(2, \mathbb{R})$ case the OPEs of the three currents $\left(H, J^{+}, J^{-}\right)$read (see, e.g., [24])

$H(w) H(0) \sim \frac{k}{w^{2}}, \quad H(w) J^{ \pm}(0) \sim \mp \frac{i \sqrt{2} J^{ \pm}(0)}{w}, \quad J^{+}(w) J^{-}(0) \sim \frac{2 k}{w^{2}}-\frac{2 i \sqrt{2} H(0)}{w}$.

From (2.36) we conclude that: (i) the two-point functions are

$$
\left\langle H\left(w_{1}\right) H\left(w_{2}\right)\right\rangle=\frac{k}{w_{12}^{2}}, \quad\left\langle J^{+}\left(w_{1}\right) J^{-}\left(w_{2}\right)\right\rangle=\frac{2 k}{w_{12}^{2}},
$$

(ii) the only non-vanishing three-point function is

$$
\left\langle H\left(w_{1}\right) J^{+}\left(w_{2}\right) J^{-}\left(w_{3}\right)\right\rangle=-2 \sqrt{2} \frac{i k}{w_{12} w_{13} w_{23}},
$$

and (iii) the non-trivial four-point functions are

$$
\begin{aligned}
\left\langle J^{+}\left(w_{1}\right) J^{-}\left(w_{2}\right) J^{+}\left(w_{3}\right) J^{-}\left(w_{4}\right)\right\rangle & =4 k^{2}\left(\frac{1}{w_{23}^{2} w_{14}^{2}}+\frac{1}{w_{12}^{2} w_{34}^{2}}\right)-\frac{8 k}{w_{12} w_{23} w_{14} w_{34}} \\
\left\langle J^{+}\left(w_{1}\right) J^{-}\left(w_{2}\right) H\left(w_{3}\right) H\left(w_{4}\right)\right\rangle & =2 k^{2} \frac{1}{w_{12}^{2} w_{34}^{2}}-\frac{4 k}{w_{13} w_{14} w_{23} w_{24}} .
\end{aligned}
$$

These four-point functions are non-trivial in the sense that in addition to the $k^{2}$ contribution they also have a term linear in $k$ (cf. (1.6)). ${ }^{8}$ Comparing to boundary correlators discussed above, the $k^{2}$ term is a counterpart with disconnected $\mathrm{AdS}_{2}$ Witten diagram contribution, while the order $k$ term corresponds to connected exchange and contact contributions due to non-trivial bulk interactions. In fact, it is possible to establish the precise matching between the tree-level 2-point and 3-point boundary correlators in (2.14), (2.18) and the current correlators $(2.37),(2.38)$ restricted to the real line using the following identification (cf. (1.2))

$$
\Phi=\kappa H, \quad \Psi=\kappa J^{+}, \quad \widetilde{\Psi}=\kappa J^{-}, \quad \kappa=\sqrt{\frac{2}{k}}=\frac{b}{\sqrt{2}} .
$$

A non-trivial consistency check is that the connected 4-point boundary correlators (2.26), (2.36) then also match with the non-trivial order $k$ parts of the 4-current correlators in (2.39). The fact that there is just a single universal proportionality coefficient $\kappa$ follows from the global group symmetry of the WZW model (this is true not only at the tree level but also to all orders in $1 / k)$.

\footnotetext{
${ }^{8}$ For instance, a four-point function which is non-vanishing but trivial in the above sense is

$$
\left\langle H\left(w_{1}\right) H\left(w_{2}\right) H\left(w_{3}\right) H\left(w_{4}\right)\right\rangle=\frac{k^{2}}{4}\left(\frac{1}{w_{12}^{2} w_{34}^{2}}+\frac{1}{w_{13}^{2} w_{24}^{2}}+\frac{1}{w_{14}^{2} w_{23}^{2}}\right) .
$$
}




\subsection{Boundary correlators in $\mathrm{PCM}_{q}$ on $\mathrm{AdS}_{2}$}

Let us now go back to the $\mathrm{SL}(2, \mathbb{R})$ principal chiral model with a general coefficient $q$ of the WZ term in (2.4) to emphasize that its boundary correlators have a complicated structure already at the tree level (containing, in particular, logarithmic terms found also in a similar $\sigma$-model context in $[1,2])$. In contrast to the Liouville and Toda theories discussed in $[6,7,10]$ here the classical conformal symmetry of $\operatorname{PCM}_{q}(2.4)$ is not enough to sufficiently constrain the boundary correlators. ${ }^{9}$ The correlators simplify precisely at the WZW point $q^{2}=1$ and this may be attributed to the emerging KM symmetry (that implies chiral decomposition in flat space).

The action (2.4) in $\mathrm{AdS}_{2}$ expanded in powers of $b$ reads (cf. $\left.(2.6)\right)^{10}$

$$
\begin{array}{r}
S=\int \mathrm{d}^{2} w\left(\partial \phi \bar{\partial} \phi+\frac{1+q}{2} e^{b \phi} \partial \psi \bar{\partial} \tilde{\psi}+\frac{1-q}{2} e^{b \phi} \bar{\partial} \psi \partial \tilde{\psi}\right) \\
=\int \mathrm{d}^{2} w\left(\partial \phi \bar{\partial} \phi+\partial \psi \bar{\partial} \tilde{\psi}+\frac{b(1+q)}{2} \phi \partial \psi \bar{\partial} \tilde{\psi}+\frac{b(1-q)}{2} \phi \bar{\partial} \psi \partial \tilde{\psi}\right. \\
\left.+\frac{b^{2}(1+q)}{8} \phi^{2} \partial \psi \bar{\partial} \tilde{\psi}+\frac{b^{2}(1-q)}{8} \phi^{2} \bar{\partial} \psi \partial \tilde{\psi}+\cdots\right) .
\end{array}
$$

Repeating the calculation of the tree-level four-point $\left\langle\Psi^{2} \widetilde{\Psi}^{2}\right\rangle$ correlator we find (cf. (2.20))

$$
\begin{aligned}
\left\langle\Psi\left(\mathrm{t}_{1}\right) \widetilde{\Psi}\left(\mathrm{t}_{2}\right) \Psi\left(\mathrm{t}_{3}\right) \widetilde{\Psi}\left(\mathrm{t}_{4}\right)\right\rangle= & \mathcal{A}_{4}\left(\mathrm{t}_{1}, \mathrm{t}_{2}, \mathrm{t}_{3}, \mathrm{t}_{4}\right)+\mathcal{A}_{4}\left(\mathrm{t}_{1}, \mathrm{t}_{4}, \mathrm{t}_{3}, \mathrm{t}_{2}\right) \\
\mathcal{A}_{4}\left(\mathrm{t}_{1}, \mathrm{t}_{2}, \mathrm{t}_{3}, \mathrm{t}_{4}\right)= & \left(\frac{1+q}{2}\right)^{2} A_{4}\left(\mathrm{t}_{1}, \mathrm{t}_{2}, \mathrm{t}_{3}, \mathrm{t}_{4}\right)+\left(\frac{1-q}{2}\right)^{2} A_{4}\left(\mathrm{t}_{2}, \mathrm{t}_{1}, \mathrm{t}_{4}, \mathrm{t}_{3}\right) \\
& +\frac{1-q^{2}}{4}\left[A_{4}\left(\mathrm{t}_{2}, \mathrm{t}_{1}, \mathrm{t}_{3}, \mathrm{t}_{4}\right)+A_{4}\left(\mathrm{t}_{1}, \mathrm{t}_{2}, \mathrm{t}_{4}, \mathrm{t}_{3}\right)\right]
\end{aligned}
$$

where $A_{4}$ is given by $(2.21),(2.22),(2.25)$. Similarly, for the $\left\langle\Phi^{2} \Psi \widetilde{\Psi}\right\rangle$ correlator we get

$$
\begin{aligned}
\left\langle\Phi\left(\mathrm{t}_{1}\right) \Phi\left(\mathrm{t}_{2}\right) \Psi\left(\mathrm{t}_{3}\right) \widetilde{\Psi}\left(\mathrm{t}_{4}\right)\right\rangle & =\mathcal{B}_{4}\left(\mathrm{t}_{1}, \mathrm{t}_{2}, \mathrm{t}_{3}, \mathrm{t}_{4}\right)+\mathcal{B}_{4}\left(\mathrm{t}_{2}, \mathrm{t}_{1}, \mathrm{t}_{3}, \mathrm{t}_{4}\right)+\mathrm{C}_{4}\left(\mathrm{t}_{1}, \mathrm{t}_{2}, \mathrm{t}_{3}, \mathrm{t}_{4}\right), \\
\mathcal{B}_{4}\left(\mathrm{t}_{1}, \mathrm{t}_{2}, \mathrm{t}_{3}, \mathrm{t}_{4}\right) & =\left(\frac{1+q}{2}\right)^{2} B_{4}\left(\mathrm{t}_{1}, \mathrm{t}_{2}, \mathrm{t}_{3}, \mathrm{t}_{4}\right)+\left(\frac{1-q}{2}\right)^{2} B_{4}\left(\mathrm{t}_{1}, \mathrm{t}_{2}, \mathrm{t}_{4}, \mathrm{t}_{3}\right), \\
\mathcal{C}_{4}\left(\mathrm{t}_{1}, \mathrm{t}_{2}, \mathrm{t}_{3}, \mathrm{t}_{4}\right) & =\frac{1+q}{2} C_{4}\left(\mathrm{t}_{1}, \mathrm{t}_{2}, \mathrm{t}_{3}, \mathrm{t}_{4}\right)+\frac{1-q}{2} C_{4}\left(\mathrm{t}_{1}, \mathrm{t}_{2}, \mathrm{t}_{4}, \mathrm{t}_{3}\right),
\end{aligned}
$$

where $B_{4}$ and $C_{4}$ are given by (2.29), (2.30), (2.33), (2.34). We conclude that these fourpoint functions contain logarithmic terms. These cancel only at the WZW point $q^{2}=1$ allowing one to relate these boundary correlators to the "connected" part of the correlators of the chiral WZW currents as explained above.

\footnotetext{
${ }^{9}$ As we remarked in the Introduction, this may be related to the fact that the $\sigma$-model fields transform as scalars (i.e. trivially) under the conformal group.

${ }^{10}$ While the $\mathrm{AdS}_{2}$ conformal factor decouples at the tree level, this will no longer be so at the quantum level as this model will have UV divergences and thus conformal anomaly (assuming reparametrizationcovariant regularization) unless $q^{2}=1$.
} 


\section{Tree-level boundary correlators in generic $\sigma$-model on $\mathrm{AdS}_{2}$}

Let us now consider the $\mathrm{AdS}_{2}$ boundary correlators in a general $\sigma$-model expanded near a constant background. This includes, in particular, the case of a WZW model for a general group $G$. We shall again demonstrate the cancellation of the logarithmic terms in the four-point correlators at the WZW point and match them with the connected part of the correlators of the chiral currents (1.6) restricted the real line.

\subsection{Action}

Let us start with a general bosonic $\sigma$-model with coupling functions $\left(G_{a b}, B_{a b}\right)$ and expand it in normal coordinates near the origin using $G_{a b}(X)=\delta_{a b}-\frac{1}{3} R_{a c b d}(0) X^{c} X^{d}+\mathcal{O}\left(X^{3}\right)$ and $B_{a b}(X)=B_{a b}(0)+\frac{1}{3} H_{a b c}(0) X^{c}+\mathcal{O}\left(X^{2}\right)$. Then its Euclidean action may be written as ${ }^{11}$

$$
\begin{aligned}
S & =\frac{1}{4 \pi} \int d^{2} x \sqrt{\mathrm{g}}\left[\mathrm{g}^{\mu \nu} G_{a b}(X)+i \epsilon^{\mu \nu} B_{a b}(X)\right] \partial_{\mu} X^{a} \partial_{\nu} X^{b} \\
& =\frac{1}{4 \pi} \int d^{2} x \sqrt{\mathrm{g}}\left[\mathrm{g}^{\mu \nu}\left(\delta_{a b}-\frac{1}{3} R_{a c b d}(0) X^{c} X^{d}+\cdots\right)+\frac{i}{3} \epsilon^{\mu \nu}\left(H_{a b c}(0) X^{c}+\cdots\right)\right] \partial_{\mu} X^{a} \partial_{\nu} X^{b} .
\end{aligned}
$$

In what follows we will consider the leading terms in this action parametrized as

$$
S=\frac{1}{4 \pi} \int d^{2} x \sqrt{\mathrm{g}}\left(\partial_{\mu} X^{a} \partial^{\mu} X^{a}-P_{a b c d} X^{a} X^{c} \partial_{\mu} X^{b} \partial^{\mu} X^{d}+i Q_{a b c} \epsilon^{\mu \nu} X^{a} \partial_{\mu} X^{b} \partial_{\nu} X^{c}+\cdots\right),
$$

where the constant real coupling functions $P$ and $Q$ are given by

$$
P_{a b c d} \equiv \frac{1}{3} R_{a b c d}(0), \quad Q_{a b c} \equiv \frac{1}{3} H_{a b c}(0) .
$$

Thus $Q$ is totally antisymmetric and $P$ has algebraic symmetries of the curvature

$$
\begin{aligned}
P_{a b c d} & =-P_{b a c d}, \quad P_{a b c d}=-P_{a b d c}, \quad P_{a b c d}=P_{c d a b}, \\
P_{a b c d}+P_{a c d b}+P_{a d b c} & =0 .
\end{aligned}
$$

To account for the manifest symmetry of the 4-vertex in $(3.2)$ in $(a, c)$ it is useful to introduce also the corresponding symmetrization of $P_{a b c d}$

$$
\begin{aligned}
\tilde{P}_{a b c d} & \equiv \frac{1}{2}\left(P_{a b c d}+P_{c b a d}\right)=-\frac{1}{2}\left(P_{b a c d}+P_{b c a d}\right)=\frac{1}{2}\left(P_{b a d c}+P_{d a b c}\right), \\
\tilde{P}_{a b c d} & =\tilde{P}_{b a d c}, \quad \tilde{P}_{a b c d}=\tilde{P}_{c b a d}, \quad \tilde{P}_{a b c d}=\tilde{P}_{a d c b} .
\end{aligned}
$$

Then specifying to the $\mathrm{AdS}_{2}$ background the action (3.2) may be written as (cf. (2.6))

$$
S=\int \mathrm{d}^{2} w\left(\partial X^{a} \bar{\partial} X^{a}-\tilde{P}_{a b c d} X^{a} X^{c} \partial X^{b} \bar{\partial} X^{d}+Q_{a b c} X^{a} \partial X^{b} \bar{\partial} X^{c}+\cdots\right) .
$$

\footnotetext{
${ }^{11}$ We ignore the overall coupling factor or $\frac{1}{\alpha^{\prime}}$ that can be absorbed into a rescaling of $X^{a}$ and then appears in $R$ and $H$.
} 
The action (3.2) represents as a particular case the expansion of the $\operatorname{PCM}_{q}$ (2.1) for an arbitrary group $G$. Let us normalize the generators $\left\{t_{a}\right\}$ and the invariant bilinear form of the Lie algebra of $G$ as $^{12}$

$$
\left[t_{a}, t_{b}\right]=f_{a b}^{c} t_{c}, \quad \operatorname{tr}\left(t_{a} t_{b}\right)=\delta_{a b} .
$$

Then choosing the parametrization of the group field as ${ }^{13} g=e^{-i \lambda t_{a} X^{a}}$ we find that in the $\mathrm{PCM}_{q}$ case

$$
\mathrm{PCM}_{q}: \quad P_{a b c d}=\mathrm{p} f_{a b e} f_{c d e}, \quad Q_{a b c}=\mathrm{q} f_{a b c}, \quad \mathrm{p}=-\frac{1}{12} \lambda^{2}, \quad \mathrm{q}=-\frac{1}{3} i \lambda q .
$$

The WZW theory corresponds to the choice $(2.2)$, i.e. $\mathbf{q}=-\operatorname{sign}(k) \frac{1}{3} i \lambda, \quad \lambda=\sqrt{\frac{2}{|k|}}$. In what follows we shall assume that $k>0$.

\subsection{Tree-level $\mathrm{AdS}_{2}$ boundary correlation functions}

The fields $X^{a}$ in (3.8) are massless and thus, assuming the Dirichlet boundary conditions, we have (cf. (2.7))

$$
\left.X^{a}(\mathrm{t}, \mathrm{z})\right|_{\mathrm{z} \rightarrow 0}=\mathrm{z} \mathrm{X}^{a}(\mathrm{t})+\cdots .
$$

They should correspond to the boundary operators with dimension $\Delta=1$. As in (2.10), their bulk-to-bulk $\mathrm{AdS}_{2}$ propagator is given by

$$
g_{a b}\left(w, w^{\prime}\right)=\left\langle X_{a}(w) X_{b}\left(w^{\prime}\right)\right\rangle=\delta_{a b} g(\eta)=-\frac{1}{2} \delta_{a b} \log \eta\left(w, w^{\prime}\right),
$$

while the bulk-to-boundary propagator is

$$
g_{a b}^{\partial}\left(\mathrm{t} ; w^{\prime}\right)=\lim _{\mathbf{z} \rightarrow 0} \frac{1}{\mathbf{z}} g_{a b}\left(\mathrm{t}, \mathbf{z} ; \mathbf{t}^{\prime}, \mathbf{z}^{\prime}\right)=\delta_{a b} \frac{2 \mathbf{z}^{\prime}}{\left(\mathrm{t}^{\prime}-\mathrm{t}\right)^{2}+\mathrm{z}^{\prime 2}}=\delta_{a b} g^{\partial}\left(\mathrm{t}, w^{\prime}\right) .
$$

Then the boundary two-point function is (cf. (2.14))

$$
\left\langle\mathrm{X}_{a}\left(\mathrm{t}_{1}\right) \mathrm{X}_{b}\left(\mathrm{t}_{2}\right)\right\rangle=\frac{2 \delta_{a b}}{\mathrm{t}_{12}^{2}} .
$$

Starting with (3.8) it is straightforward also to compute the three-point function

$$
\left\langle\mathrm{X}_{a}\left(\mathrm{t}_{1}\right) \mathrm{X}_{b}\left(\mathrm{t}_{2}\right) \mathrm{X}_{c}\left(\mathrm{t}_{3}\right)\right\rangle=\mathrm{X}_{b}\left(\mathrm{t}_{2}\right)
$$

The connected tree-level four-point function receives contributions from both exchange diagrams and contact diagrams

$$
\left\langle\mathrm{X}_{a}\left(\mathrm{t}_{1}\right) \mathrm{X}_{b}\left(\mathrm{t}_{2}\right) \mathrm{X}_{c}\left(\mathrm{t}_{3}\right) \mathrm{X}_{d}\left(\mathrm{t}_{4}\right)\right\rangle=\mathrm{G}_{a b c d}^{\text {exch }}\left(\mathrm{t}_{1}, \mathrm{t}_{2}, \mathrm{t}_{3}, \mathrm{t}_{4}\right)+\mathrm{G}_{a b c d}^{\text {cont }}\left(\mathrm{t}_{1}, \mathrm{t}_{2}, \mathrm{t}_{3}, \mathrm{t}_{4}\right) .
$$

\footnotetext{
${ }^{12}$ We assume that $t_{a}$ are Hermitian and thus the structure constants $f_{a b}{ }^{c}$ are purely imaginary. The group indices are raised or lowered by $\delta_{a b}$, implying that $f_{a b c}=f_{a b}{ }^{c}$ is fully anti-symmetric. Repeated group indices are summed over, regardless of their positions.

${ }^{13}$ Notice that this parametrization is different from $(2.3)$ used in the $\operatorname{SL}(2, \mathbb{R})$ case.
} 
Exchange diagrams. The exchange part contains contributions of the three different channels

$$
\mathrm{G}_{a b c d}^{\text {exch }}\left(\mathrm{t}_{1}, \mathrm{t}_{2}, \mathrm{t}_{3}, \mathrm{t}_{4}\right)=G_{a b c d}^{\text {exch }}\left(\mathrm{t}_{1}, \mathrm{t}_{2}, \mathrm{t}_{3}, \mathrm{t}_{4}\right)+G_{a c b d}^{\text {exch }}\left(\mathrm{t}_{1}, \mathrm{t}_{3}, \mathrm{t}_{2}, \mathrm{t}_{4}\right)+G_{a d c b}^{\text {exch }}\left(\mathrm{t}_{1}, \mathrm{t}_{4}, \mathrm{t}_{3}, \mathrm{t}_{2}\right) .
$$

As these are related by permutations (crossing), we only need to compute one of them

$$
G_{a b c d}^{\mathrm{exch}}\left(\mathrm{t}_{1}, \mathrm{t}_{2}, \mathrm{t}_{3}, \mathrm{t}_{4}\right)=Q_{a b e} Q_{c d e} \overbrace{\ldots}
$$

Note that using integration by parts, one can always arrange so that the derivatives in the cubic vertex in (3.8) act only on the two external legs. ${ }^{14}$ Then the 6 terms in the cubic vertex can be written as

$$
K\left(\mathrm{t}_{1}, \mathrm{t}_{2}, w\right)=3 \partial g^{\partial}\left(\mathrm{t}_{1}, w\right) \bar{\partial} g^{\partial}\left(\mathrm{t}_{2}, w\right)-3 \bar{\partial} g^{\partial}\left(\mathrm{t}_{1}, w\right) \partial g^{\partial}\left(\mathrm{t}_{2}, w\right),
$$

where the two terms arise from the two ways of acting by derivative on the external legs and the factor of 3 comes from rearranging other similar cubic terms. Using (3.19), we find for the exchange diagram

$$
\begin{aligned}
G_{a b c d}^{\text {exch }}\left(\mathrm{t}_{1}, \mathrm{t}_{2}, \mathrm{t}_{3}, \mathrm{t}_{4}\right) & =Q_{a b e} Q_{c d e} \int \mathrm{d}^{2} w \mathrm{~d}^{2} w^{\prime} K\left(\mathrm{t}_{1}, \mathrm{t}_{2}, w\right) g\left(w, w^{\prime}\right) K\left(\mathrm{t}_{3}, \mathrm{t}_{4}, w\right) \\
& =9 Q_{a b e} Q_{c d e} \mathrm{H}\left(\mathrm{t}_{1}, \mathrm{t}_{2}, \mathrm{t}_{3}, \mathrm{t}_{4}\right), \\
\mathbf{H}\left(\mathrm{t}_{1}, \mathrm{t}_{2}, \mathrm{t}_{3}, \mathrm{t}_{4}\right) & =\tilde{\mathrm{H}}\left(\mathrm{t}_{1}, \mathrm{t}_{2}, \mathrm{t}_{3}, \mathrm{t}_{4}\right)-\tilde{\mathrm{H}}\left(\mathrm{t}_{1}, \mathrm{t}_{2}, \mathrm{t}_{4}, \mathrm{t}_{3}\right)-\tilde{\mathrm{H}}\left(\mathrm{t}_{2}, \mathrm{t}_{1}, \mathrm{t}_{3}, \mathrm{t}_{4}\right)+\tilde{\mathrm{H}}\left(\mathrm{t}_{2}, \mathrm{t}_{1}, \mathrm{t}_{4}, \mathrm{t}_{3}\right) .
\end{aligned}
$$

Here $\tilde{\mathbf{H}}$ was defined in (2.22) and computed in (2.25).

Contact diagrams. Since the quartic vertex in (3.8) contains derivatives, the contact contribution may also be represented as a sum of the three contributions

$$
\mathrm{G}_{a b c d}^{\mathrm{cont}}\left(\mathrm{t}_{1}, \mathrm{t}_{2}, \mathrm{t}_{3}, \mathrm{t}_{4}\right)=G_{a b c d}^{\mathrm{cont}}\left(\mathrm{t}_{1}, \mathrm{t}_{2}, \mathrm{t}_{3}, \mathrm{t}_{4}\right)+G_{a c b d}^{\mathrm{cont}}\left(\mathrm{t}_{1}, \mathrm{t}_{3}, \mathrm{t}_{2}, \mathrm{t}_{4}\right)+G_{a b d c}^{\mathrm{cont}}\left(\mathrm{t}_{1}, \mathrm{t}_{2}, \mathrm{t}_{4}, \mathrm{t}_{3}\right)
$$

\footnotetext{
${ }^{14}$ Note that $X^{a}\left(\partial X^{b} \bar{\partial} X^{c}-\partial X^{c} \bar{\partial} X^{b}\right)$ is fully anti-symmetric in $a, b, c$ up to a total derivative.
} 
Here we have indicated explicitly the coupling tensors appearing from each diagram (the factor of 2 arises from two ways of contracting the two legs without derivative). Explicitly, we get

$$
\begin{aligned}
G_{a b c d}^{\mathrm{cont}}\left(\mathrm{t}_{1}, \mathrm{t}_{2}, \mathrm{t}_{3}, \mathrm{t}_{4}\right)= & 2 \tilde{P}_{a b c d}\left[\int \mathrm{d}^{2} w \partial g\left(\mathrm{t}_{1}, w\right) g\left(\mathrm{t}_{2}, w\right) \bar{\partial} g\left(\mathrm{t}_{3}, w\right) g\left(\mathrm{t}_{4}, w\right)+\left(\mathrm{t}_{1} \leftrightarrow \mathrm{t}_{3}\right)\right] \\
& +2 \tilde{P}_{a b c d}\left[\int \mathrm{d}^{2} w g\left(\mathrm{t}_{1}, w\right) \partial g\left(\mathrm{t}_{2}, w\right) g\left(\mathrm{t}_{3}, w\right) \bar{\partial} g\left(\mathrm{t}_{4}, w\right)+\left(\mathrm{t}_{2} \leftrightarrow \mathrm{t}_{4}\right)\right],
\end{aligned}
$$

where we used (3.7). As a result,

$$
G_{a b c d}^{\mathrm{cont}}\left(\mathrm{t}_{1}, \mathrm{t}_{2}, \mathrm{t}_{3}, \mathrm{t}_{4}\right)=4 \tilde{P}_{a b c d} \mathrm{I}\left(\mathrm{t}_{1}, \mathrm{t}_{2}, \mathrm{t}_{3}, \mathrm{t}_{4}\right)
$$

where

$$
\begin{aligned}
\left.\mathrm{I}_{(}, \mathrm{t}_{1}, \mathrm{t}_{2}, \mathrm{t}_{3}, \mathrm{t}_{4}\right) & \equiv \int \mathrm{d}^{2} w \partial g\left(\mathrm{t}_{1}, w\right) g\left(\mathrm{t}_{2}, w\right) \bar{\partial} g\left(\mathrm{t}_{3}, w\right) g\left(\mathrm{t}_{4}, w\right)+\left(\mathrm{t}_{1} \leftrightarrow \mathrm{t}_{3}\right) \\
& =\int \mathrm{d}^{2} w g\left(\mathrm{t}_{1}, w\right) \partial g\left(\mathrm{t}_{2}, w\right) g\left(\mathrm{t}_{3}, w\right) \bar{\partial} g\left(\mathrm{t}_{4}, w\right)+\left(\mathrm{t}_{2} \leftrightarrow \mathrm{t}_{4}\right) \\
& =\frac{1}{\mathrm{t}_{14}^{2} \mathrm{t}_{23}^{2}} \log \left(\frac{\mathrm{t}_{12} \mathrm{t}_{34}}{\mathrm{t}_{13} \mathrm{t}_{24}}\right)^{2}+\frac{1}{\mathrm{t}_{12}^{2} \mathrm{t}_{34}^{2}} \log \left(\frac{\mathrm{t}_{14} \mathrm{t}_{23}}{\mathrm{t}_{13} \mathrm{t}_{24}}\right)^{2}+\frac{2}{\mathrm{t}_{12} \mathrm{t}_{14} \mathrm{t}_{23} \mathrm{t}_{34}} .
\end{aligned}
$$

Collecting the contributions in (3.17), (3.20) and (3.25), (3.26), the four-point function in (3.16) may be written as

$$
\begin{aligned}
\left\langle\mathrm{X}_{a}\left(\mathrm{t}_{1}\right) \mathrm{X}_{b}\left(\mathrm{t}_{2}\right) \mathrm{X}_{c}\left(\mathrm{t}_{3}\right) \mathrm{X}_{d}\left(\mathrm{t}_{4}\right)\right\rangle= & 4 \alpha_{s} \mathrm{l}\left(\mathrm{t}_{1}, \mathrm{t}_{2}, \mathrm{t}_{3}, \mathrm{t}_{4}\right)+4 \alpha_{t} \mathrm{l}\left(\mathrm{t}_{1}, \mathrm{t}_{3}, \mathrm{t}_{2}, \mathrm{t}_{4}\right)+4 \alpha_{u} \mathrm{l}\left(\mathrm{t}_{1}, \mathrm{t}_{2}, \mathrm{t}_{4}, \mathrm{t}_{3}\right) \\
& +9 \beta_{s} \mathrm{H}\left(\mathrm{t}_{1}, \mathrm{t}_{2}, \mathrm{t}_{3}, \mathrm{t}_{4}\right)+9 \beta_{t} \mathrm{H}\left(\mathrm{t}_{1}, \mathrm{t}_{3}, \mathrm{t}_{2}, \mathrm{t}_{4}\right)+9 \beta_{u} \mathrm{H}\left(\mathrm{t}_{1}, \mathrm{t}_{4}, \mathrm{t}_{3}, \mathrm{t}_{2}\right),
\end{aligned}
$$

where in the r.h.s. we suppressed the indices $(a, b, c, d)$ introducing the symbolic notation $(s, t, u \text { stand for different channels })^{15}$

$$
\begin{array}{lll}
\alpha_{s}=\tilde{P}_{a b c d}, & \alpha_{t}=\tilde{P}_{a c b d}, & \alpha_{u}=\tilde{P}_{a b d c}, \\
\beta_{s}=Q_{a b e} Q_{c d e}, & \beta_{t}=Q_{a c e} Q_{b d e}, & \beta_{u}=Q_{a d e} Q_{c b e} .
\end{array}
$$

Using the expressions for the integrals $\mathrm{H}$ in (3.21), (2.25) and I in (3.26) one can compute the four-point function (3.27) explicitly.

We find that the logarithmic terms in (3.27) cancel if the following relations are satisfied

$$
\beta_{s}=\frac{8}{9}\left(\alpha_{s}-\alpha_{u}\right), \quad \beta_{t}=\frac{8}{9}\left(\alpha_{t}-\alpha_{u}\right), \quad \beta_{u}=\frac{8}{9}\left(\alpha_{s}-\alpha_{t}\right) .
$$

This implies that

$$
\beta_{s}-\beta_{t}-\beta_{u}=Q_{a b e} Q_{c d e}-Q_{a c e} Q_{b d e}-Q_{a d e} Q_{c b e}=Q_{a b e} Q_{c d e}+Q_{c a e} Q_{b d e}+Q_{a d e} Q_{b c e}=0,
$$

\footnotetext{
${ }^{15}$ Note that the permutations of legs on the first and second lines of (3.27) are different.
} 
and also that

$$
\frac{9}{4} Q_{a b e} Q_{c d e}=\tilde{P}_{a b c d}-\tilde{P}_{a b d c}=P_{a b c d}+P_{c b a d}-P_{a b d c}-P_{d b a c}=3 P_{a b c d}, \quad \text { i.e. } P_{a b c d}=\frac{3}{4} Q_{a b e} Q_{c d e},
$$

where we used (3.6) and symmetry properties of the curvature tensor in (3.4) and (3.5). Written in terms of $R$ and $H$ in (3.3) this reads

$$
R_{a b c d}=\frac{1}{4} H_{a b e} H_{c d e} .
$$

Interestingly, the trace of this relation, i.e. $R_{a c}=\frac{1}{4} H_{a b e} H_{c b e}$, is the same as the vanishing of the one-loop beta-function [25] of the $\sigma$-model in (3.1).

In the group space case (3.10) the condition (3.31) is automatically satisfied due to the Jacobi identity for the structure constants. The condition (3.32) or (3.33) reduces to

$$
\mathrm{q}^{2}=\frac{4}{3} \mathrm{p}, \quad \text { i.e. } \quad q^{2}=1,
$$

i.e. is valid only in the WZW model case (cf. (2.2), (3.10)).

\subsection{WZW model case: matching with correlators of chiral currents}

Thus the cancellation of the logarithmic terms in the four-point boundary correlators of a generic $\sigma$-model in $\mathrm{AdS}_{2}$ happens only in the WZW model. This generalizes the observation made in section 2 in the $\mathrm{SL}(2, \mathbb{R}) \mathrm{WZW}$ case. Then the resulting expression for the connected four-point correlator (3.27) may be written as (using (3.10) with $q=1$, i.e. $\left.\lambda^{2}=\frac{2}{k}\right)$

$$
\left\langle\mathrm{X}_{a}\left(\mathrm{t}_{1}\right) \mathrm{X}_{b}\left(\mathrm{t}_{2}\right) \mathrm{X}_{c}\left(\mathrm{t}_{3}\right) \mathrm{X}_{d}\left(\mathrm{t}_{4}\right)\right\rangle=\frac{4}{k}\left(\frac{f_{a b e} f_{c d e}}{\mathrm{t}_{12} \mathrm{t}_{13} \mathrm{t}_{23} \mathrm{t}_{34}}+\frac{f_{a c e} f_{d b e}}{\mathrm{t}_{12} \mathrm{t}_{13} \mathrm{t}_{23} \mathrm{t}_{24}}+\frac{f_{a d e} f_{b c e}}{\mathrm{t}_{12} \mathrm{t}_{13} \mathrm{t}_{14} \mathrm{t}_{23}}\right) .
$$

As in the $\mathrm{SL}(2, \mathbb{R})$ case (cf. (2.26), (2.36)), we can now explicitly check the correspondence between $\mathrm{AdS}_{2}$ boundary correlators and holomorphic correlation functions of Kac-Moody currents.

The basic OPE relation for the WZW chiral currents on the plane (1.3) gives the two-point function (1.4). Higher point correlators can be obtained by repeatedly using the OPE (1.3). ${ }^{16}$ In particular, one finds

$$
\begin{aligned}
\left\langle J_{a}\left(w_{1}\right) J_{b}\left(w_{2}\right) J_{c}\left(w_{3}\right)\right\rangle & =\frac{k f_{a b c}}{w_{12} w_{13} w_{23}}, \\
\left\langle J_{a}\left(w_{1}\right) J_{b}\left(w_{2}\right) J_{c}\left(w_{3}\right) J_{d}\left(w_{4}\right)\right\rangle & =\frac{k^{2} \delta_{a b} \delta_{c d}}{w_{12}^{2} w_{34}^{2}}+\frac{k f_{a b e} f_{c d e}}{w_{12} w_{34} w_{23} w_{24}}+\left(\begin{array}{c}
w_{2} \leftrightarrow w_{3} \\
b \leftrightarrow c
\end{array}\right)+\left(\begin{array}{c}
w_{2} \leftrightarrow w_{4} \\
b \leftrightarrow d
\end{array}\right) .
\end{aligned}
$$

\footnotetext{
${ }^{16}$ The current-current OPE translates into the (recursion) relation [26]

$\left\langle J_{a_{1}}\left(w_{1}\right) \ldots J_{a_{n}}\left(w_{n}\right)\right\rangle=\sum_{j=2}^{n}\left\langle J_{a_{2}}\left(w_{2}\right) \ldots J_{a_{j-1}}\left(w_{j-1}\right)\left[\frac{k \delta_{a_{1} a_{j}}}{\left(w_{1}-w_{j}\right)^{2}}+\frac{f_{a_{1} a_{j} c} J_{c}\left(w_{j}\right)}{w_{1}-w_{j}}\right] J_{a_{j+1}}\left(w_{j+1}\right) \ldots J_{a_{n}}\left(w_{n}\right)\right\rangle$.
}

The mutual locality of the KM currents implies a trivial (meromorphic) singularity structure and the solution of the above relation is simply obtained by isolating poles as in (3.36)-(3.38). 
The "connected" part of (3.37) may be written as

$$
\begin{aligned}
\left\langle J_{a}\left(w_{1}\right) J_{b}\left(w_{2}\right) J_{c}\left(w_{3}\right) J_{d}\left(w_{4}\right)\right\rangle_{\mathrm{conn}} & =k\left(\frac{f_{\text {abe }} f_{c d e}}{w_{12} w_{34} w_{23} w_{24}}+\frac{f_{\text {ace }} f_{\text {dbe }}}{w_{13} w_{24} w_{23} w_{34}}+\frac{f_{\text {ade }} f_{\text {bce }}}{w_{14} w_{23} w_{43} w_{24}}\right) \\
& =k\left(\frac{f_{\text {abe }} f_{c d e}}{w_{12} w_{13} w_{23} w_{34}}+\frac{f_{\text {ace }} f_{d b e}}{w_{12} w_{13} w_{23} w_{24}}+\frac{f_{\text {ade }} f_{b c e}}{w_{12} w_{13} w_{14} w_{23}}\right) .
\end{aligned}
$$

Here in the second line we wrote an equivalent expression (expressing crossing symmetry of the four-point function) that is a consequence of the Jacobi identity for the structure constants.

Restricting the points to the real line $\left(w_{i} \rightarrow \mathrm{t}_{i}\right)$ we can identify the two-point (1.4) and three-point (1.5) correlators of the currents with the corresponding boundary correlators in (3.14) and (3.15) up to an overall universal factor $\kappa^{n}$ where $n=2,3, \ldots$ is the number of legs. Explicitly, this amounts to the formal identification (assuming $k>0$ )

$$
\mathrm{X}^{a} \rightarrow \kappa J^{a}, \quad \kappa=\sqrt{\frac{2}{k}} .
$$

Indeed, the two-point functions match if $\kappa^{2}=\frac{2}{k}$, while the three-point functions match for $Q_{a b c}$ in (3.15) related to $f_{a b c}$ in (3.36) as in (3.10) and $\kappa^{3}=\frac{2}{k} \lambda$. Furthermore, the four-point correlator (3.35) is also in precise agreement with the boundary restriction of the connected part of the correlator of four currents in (3.38).

As already mentioned in the Introduction, one can give a simple semiclassical argument supporting the relation (3.39), i.e. the expression for $\kappa$ that, we remark, is same as in (2.40). Starting with the expression for the $w$-component of the chiral current consistent with $k>0$ and (1.3), (3.36) (see, e.g., [19]) $J^{a}=-k \operatorname{tr}\left(t^{a} \partial g g^{-1}\right)$ and using the parametrization $g=e^{-i \lambda t_{a} X^{a}}$ we get in the $\mathbf{z} \rightarrow 0$ limit (for the boundary asymptotics in (3.11))

$$
\mathrm{z} \rightarrow 0: \quad J^{a}=i k \lambda \partial X^{a}+\cdots=i k \lambda \frac{1}{2}\left(\partial_{\mathrm{t}}-i \partial_{\mathrm{z}}\right)\left(\mathrm{z} \mathrm{X}^{a}+\cdots\right)=\frac{1}{2} k \lambda \mathrm{X}^{a}+\cdots .
$$

This suggests the identification $J^{a}=\frac{1}{2} k \lambda \mathrm{X}^{a}$ as in (3.39) where $\lambda$ is given by (2.2), i.e. $\kappa=\sqrt{\frac{2}{k}}$.

\section{Quantum corrections to boundary correlators in $\mathrm{SL}(2, \mathbb{R}) \mathrm{WZW}$ model}

The above discussion was restricted to consideration of tree-level terms in the boundary correlators in $\mathrm{AdS}_{2}$. Let us now try to test the relation between the boundary correlators of WZW fields and chiral currents (1.2) beyond the classical (large $k$ ) limit. This requires determining loop corrections to $\mathrm{AdS}_{2}$ boundary correlators. Similar computations were done in the Liouville and Toda theories in $[7,11,12]$ and it was found that the analogs of the coefficient $\kappa$ in (1.2) that relate boundary correlators of elementary fields in $\mathrm{AdS}_{2}$ to correlators of CFT currents (stress tensor and $\mathcal{W}$-symmetry currents) restricted to real line receive quantum corrections. 
In the present WZW model case, the simplicity of the semiclassical argument in (3.40) suggests instead that the relation (1.2) or (2.40), (3.39) may be exact. ${ }^{17}$

To provide support to this conjecture below we shall consider the computation of oneloop corrections to the two-point and three-point boundary correlators (1.1) on the example of the $\mathrm{SL}(2, \mathbb{R})$ WZW model. A central issue will be the choice of a UV regularization and subtraction scheme consistent with underlying $\operatorname{SL}(2, \mathbb{R})$ symmetry of the model. It turns out to be possible to relate the scheme ambiguity to the definition of the propagator at the coinciding points, i.e. to the choice of the renormalized value of the self-contraction contributions.

\subsection{One-loop corrections to the two-point correlators}

Let us start with computing the one-loop corrections to the tree-level two-point functions (2.14) for the fields in the action (2.6), i.e. to the boundary correlators $\langle\Psi \widetilde{\Psi}\rangle$ and $\langle\Phi \Phi\rangle$.

\subsection{1 $\langle\Psi \widetilde{\Psi}\rangle$}

One-loop corrections to the $\psi, \tilde{\psi}$ propagator in $\mathrm{AdS}_{2}$ come from the following diagrams:

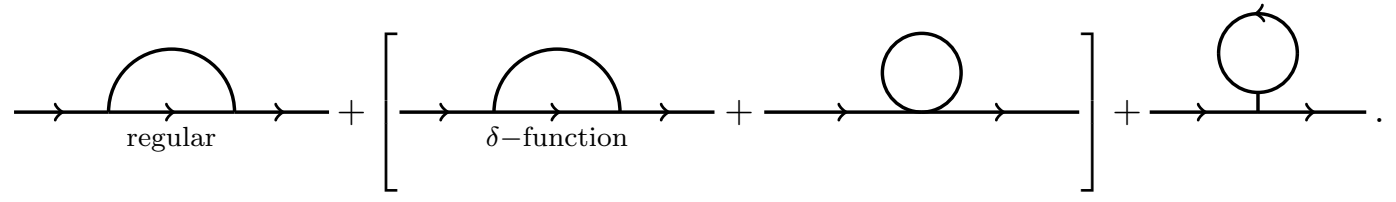

Here we have separated the contributions of the regular and $\delta$-function terms in (2.12) combining the latter with the self-contraction diagram corresponding to the vertex $\phi^{2} \psi \tilde{\psi}$ in (2.6) as both are proportional to the free scalar propagator at the coinciding points, i.e. $g(w, w)$ (cf. (2.10), (2.11)). The last tadpole diagram with a $\psi$ loop is linearly divergent and may be removed by imposing the normalization condition $\langle\phi\rangle=0$.

The first contribution in (4.1) involving the regular part of the second derivative of the propagator in (2.12) (with legs taken to the boundary) is given by

$$
\begin{aligned}
D_{\Psi \widetilde{\Psi}}\left(\mathrm{t}_{12}\right) & =\overbrace{\text { regular }}^{\mathrm{t}_{1}} \overbrace{\widehat{E}\left(\mathrm{t}_{12}\right)} \equiv \int d^{2} w d^{2} w^{\prime} \frac{1}{\left(\mathrm{t}-\mathrm{t}_{1}-i \mathrm{z}\right)^{2}} \frac{1}{\left(\mathrm{t}^{\prime}-\mathrm{t}_{2}+i \mathbf{z}^{\prime}\right)^{2}} \frac{b^{2}}{\pi^{2}} \widehat{E}\left(\mathrm{t}_{12}\right) \\
& =\int d^{2} w d^{2} w^{\prime} \frac{1}{\left.\left(\bar{w}-\mathrm{t}_{1}\right)^{2}+i\left(\mathbf{z}+\mathrm{z}^{\prime}\right)\right]^{2}} \frac{1}{\left(w^{\prime}-\mathrm{t}_{2}\right)^{2}} \frac{1}{\left(w-\bar{w}^{\prime}\right)^{2}} g\left(w, w^{\prime}\right)=\partial_{\mathrm{t}_{1}} \partial_{\mathrm{t}_{2}} J\left(\mathrm{t}_{12}\right), \\
J\left(\mathrm{t}_{12}\right) & \equiv \int d^{2} w d^{2} w^{\prime} \frac{1}{\bar{w}-\mathrm{t}_{1}} \frac{1}{w^{\prime}-\mathrm{t}_{2}} \frac{1}{\left(w-\bar{w}^{\prime}\right)^{2}} g\left(w, w^{\prime}\right) .
\end{aligned}
$$

\footnotetext{
${ }^{17}$ One could wonder if the level $k$ in (1.2) may get a familiar quantum shift by the dual Coxeter number of $G$ (i.e. $k \rightarrow k+c_{G}$ ) which is known to appear from the quantum jacobian transformation from the group fields $g$ to currents and in the Sugawara construction of the stress tensor and related computation of the central charge. As we shall argue below, there exists a natural computation scheme in which this does not apparently happen in the present case of $\kappa$ in (1.2).
} 
By formal shifting and rescaling $w, w^{\prime}$ one may try to argue that the integral $J$ should be independent of $t_{1}, t_{2}$. However, it is IR divergent and thus requires a regularization. A regularization will then be expected to give $J \sim \log \left(\Lambda^{-1}\left|t_{1}-t_{2}\right|\right)$ and thus a finite $\sim \frac{1}{t_{12}^{2}}$ contribution to $\widehat{E}\left(\mathrm{t}_{12}\right)$. Indeed, integrating by parts the formal expression in (4.4) we get (using $(2.11))^{18}$

$$
J=\int d^{2} w d^{2} w^{\prime} \frac{1}{\bar{w}-\mathrm{t}_{1}} \frac{1}{w^{\prime}-\mathrm{t}_{2}} \frac{1}{w-\bar{w}^{\prime}} \partial_{w} g\left(w, w^{\prime}\right)=\int_{0}^{1} d x \int_{0}^{\infty} d y \frac{\pi^{2}\left(1+6 i y+2 i x y-8 y^{2}\right)}{(-i+2 y)(-i+2 y+2 x y)^{2}} .
$$

This is divergent due to the contribution from the $y \rightarrow+\infty$ region where the integrand scales as $\sim 1 /\left(x^{2} y\right)$. A cutoff on the $z, z^{\prime}$ integrals near zero in (4.4) translates into the modified integration range $0<y<\frac{\Lambda}{\mathrm{t}_{12}}, \Lambda \rightarrow \infty$. Then we find for the regularized integral ${ }^{19}$

$$
J\left(\mathrm{t}_{12} ; \Lambda\right)=\int_{0}^{1} d x \int_{0}^{\Lambda / \mathrm{t}_{12}} d y \frac{\pi^{2}\left(1+6 i y-2 i x y+8 y^{2}\right)}{(-i+2 y)(-i+2 y+2 x y)^{2}} \stackrel{\Lambda \rightarrow \infty}{=}-\frac{\pi^{2}}{2} \log \frac{\Lambda}{\mathrm{t}_{12}}+\text { finite }
$$

and thus

$$
\widehat{E}\left(\mathrm{t}_{12}\right)=\partial_{\mathrm{t}_{1}} \partial_{\mathrm{t}_{2}}\left[-\frac{\pi^{2}}{2} \log \frac{\Lambda}{\mathrm{t}_{12}}+\cdots\right]=\frac{\pi^{2}}{2 \mathrm{t}_{12}^{2}} .
$$

Including also the contribution of the square bracket terms in (4.1) which depend on regularized value of $g(w, w)$ we finish with the following one-loop (i.e. order $b^{2} \sim \frac{1}{k}$ ) correction to the tree-level boundary correlator $(2.14)$

$$
\left\langle\Psi\left(\mathrm{t}_{1}\right) \widetilde{\Psi}\left(\mathrm{t}_{2}\right)\right\rangle_{1-\text { loop }}=\frac{2 b^{2}}{\mathrm{t}_{12}^{2}}\left(1-g_{0}\right), \quad g_{0} \equiv g(w, w) .
$$

Thus a particular scheme choice where $g_{0}=1$ would lead to the vanishing of the one-loop correction.

To put this in a more general context, while the WZW is UV finite in the sense that there is no coupling renormalization, there may still be a wave function renormalization (i.e. UV divergent $Z$-factor in the off-shell 2-point function). This should be accounted for in the definition of the S-matrix: the scattering amplitudes defined in terms of correlators with extra powers of $Z$ will be automatically finite (see, e.g., a discussion in [27] and refs. there). Similar considerations should apply to the analog of S-matrix in AdS (see section 5) and thus to the boundary correlators. Here we will effectively by-pass this subtlety by simply assuming a particular subtraction under which the wave-function renormalization factor is trivial. ${ }^{20}$ It remains an open question how this scheme extends in a consistent way to higher loop orders.

\subsection{2 $\langle\Phi \Phi\rangle$}

The one-loop correction to the boundary two-point function $\left\langle\Phi\left(t_{1}\right) \Phi\left(t_{2}\right)\right\rangle$ is given by the sum of two diagrams: a bubble and a self-contraction diagram.

\footnotetext{
${ }^{18}$ The integral over $z, z^{\prime}$ here may be split and turned into a double integral over $z, Z$ with $0<z<Z$. Then setting $Z=y \mathrm{t}_{12}, z=x y \mathrm{t}_{12}$ one is to integrate over $0<x<1$ and $0<y<\infty$.

${ }^{19}$ We first integrate over $x$ and then add and subtract the leading term of the $y \rightarrow \infty$ expansion.

${ }^{20}$ For some recent discussions of one-loop self-energy corrections in AdS see [28-30].
} 
Bubble. The bubble contribution is

$$
\mathrm{t}_{1} \mathrm{t}_{2}=4 \frac{b^{2}}{\pi^{2}}\left[\widehat{D}\left(\mathrm{t}_{12}\right)+\pi \widehat{D}_{+}\left(\mathrm{t}_{12}\right)+\pi \widehat{D}_{-}\left(\mathrm{t}_{12}\right)+\pi^{2} \widehat{D}_{\text {cont }}\left(\mathrm{t}_{12}\right)\right] .
$$

Here we decomposed the derivatives of both propagators (2.12) in the loop into the regular and $\delta$-function parts getting four terms: with no $\delta$-function factors $(\widehat{D})$, with one $\left(\widehat{D}_{ \pm}\right)$ and with two $\left(\widehat{D}_{\text {cont }}\right)$. Explicitly,

$$
\begin{aligned}
\widehat{D}\left(\mathrm{t}_{12}\right) & =\int d^{2} w d^{2} w^{\prime} \frac{\mathrm{z}}{\left(\mathrm{t}-\mathrm{t}_{1}\right)^{2}+\mathrm{z}^{2}} \frac{\mathrm{z}^{\prime}}{\left(\mathrm{t}^{\prime}-\mathrm{t}_{2}\right)^{2}+\mathrm{z}^{\prime 2}} \frac{1}{\left[\left(\mathrm{t}-\mathrm{t}^{\prime}\right)^{2}+\left(\mathrm{z}+\mathrm{z}^{\prime}\right)^{2}\right]^{2}} \\
\widehat{D}_{ \pm}\left(\mathrm{t}_{12}\right) & =\int d^{2} w d^{2} w^{\prime} \frac{\mathrm{z}}{\left(\mathrm{t}-\mathrm{t}_{1}\right)^{2}+\mathrm{z}^{2}} \frac{\mathrm{z}^{\prime}}{\left(\mathrm{t}^{\prime}-\mathrm{t}_{2}\right)^{2}+\mathrm{z}^{\prime 2}} \frac{1}{\left[\mathrm{t}-\mathrm{t}^{\prime} \pm i\left(\mathrm{z}+\mathrm{z}^{\prime}\right)\right]^{2}} \delta\left(w-w^{\prime}\right) \\
& =-\frac{1}{4} \int d^{2} w \frac{1}{\left(\mathrm{t}-\mathrm{t}_{1}\right)^{2}+\mathrm{z}^{2}} \frac{1}{\left(\mathrm{t}-\mathrm{t}_{2}\right)^{2}+\mathrm{z}^{2}}
\end{aligned}
$$

Integrating over $t, t^{\prime}, z^{\prime}$ gives

$$
\widehat{D}\left(\mathrm{t}_{12}\right)=\frac{\pi^{2}}{2} \int_{0}^{\infty} d \mathrm{z} \frac{1}{\mathrm{z}\left(\mathrm{t}_{12}^{2}+4 \mathrm{z}^{2}\right)}, \quad \widehat{D}_{ \pm}\left(\mathrm{t}_{12}\right)=-\frac{\pi}{2} \int_{0}^{\infty} d \mathrm{z} \frac{1}{\mathrm{z}\left(\mathrm{t}_{12}^{2}+4 \mathrm{z}^{2}\right)}=-\frac{1}{\pi} \widehat{D}\left(\mathrm{t}_{12}\right) .
$$

Self-contraction. With the same decomposition of the two derivatives of the propagator in the loop (2.12) we get

$$
\mathrm{t}_{1} \mathrm{t}_{2}=-4 \frac{b^{2}}{\pi}\left[\widehat{D}_{ \pm}\left(\mathrm{t}_{12}\right)+\pi \widehat{D}_{\text {cont }}\left(\mathrm{t}_{12}\right)\right] .
$$

As a result, $\widehat{D}_{\text {cont }}$ here exactly cancels against the double $\delta$-function part in the bubble diagram (4.9). The total expression for the one-loop correction is then

$$
\langle\Phi \Phi\rangle_{1-\text { loop }}=\frac{4 b^{2}}{\pi^{2}}\left(\widehat{D}+2 \pi \widehat{D}_{ \pm}\right)-\frac{4 b^{2}}{\pi} \widehat{D}_{ \pm}=\frac{4 b^{2}}{\pi^{2}}\left(\widehat{D}+\pi \widehat{D}_{ \pm}\right)=0,
$$

where we used (4.11), i.e. $\widehat{D}_{ \pm}=-\frac{1}{\pi} \widehat{D}$.

A more rigorous derivation of (4.13) requires introducing a regularization factor $z^{\varepsilon}$ in each (formally divergent) AdS integral. Then

$$
\begin{aligned}
\widehat{D}_{\varepsilon}\left(\mathrm{t}_{12}\right) & =\int d^{2} w d^{2} w^{\prime} \mathrm{z}^{\varepsilon} \mathrm{z}^{\prime \varepsilon} \frac{\mathrm{z}}{\left(\mathrm{t}-\mathrm{t}_{1}\right)^{2}+\mathrm{z}^{2}} \frac{\mathrm{z}^{\prime}}{\left(\mathrm{t}^{\prime}-\mathrm{t}_{2}\right)^{2}+\mathrm{z}^{\prime 2}} \frac{1}{\left[\left(\mathrm{t}-\mathrm{t}^{\prime}\right)^{2}+\left(\mathrm{z}+\mathrm{z}^{\prime}\right)^{2}\right]^{2}} \\
& =\frac{1}{\mathrm{t}_{12}^{2-2 \varepsilon}} \frac{\pi^{3} 4^{-\varepsilon}(2 \varepsilon+1) \cot (\pi \varepsilon) \Gamma(-2 \varepsilon-2) \Gamma(\varepsilon+2)}{\Gamma(-\varepsilon)} \\
\widehat{D}_{ \pm, \varepsilon}\left(\mathrm{t}_{12}\right) & =-\frac{1}{4} \int d^{2} w \mathrm{z}^{2 \varepsilon} \frac{1}{\left(\mathrm{t}-\mathrm{t}_{1}\right)^{2}+\mathrm{z}^{2}} \frac{1}{\left(\mathrm{t}-\mathrm{t}_{2}\right)^{2}+\mathrm{z}^{2}}=-\frac{1}{\mathrm{t}_{12}^{2-2 \varepsilon}} 2^{-2-2 \varepsilon} \pi^{2} \csc (\pi \varepsilon) .
\end{aligned}
$$


Then expanding for small $\varepsilon$ gives

$$
\widehat{D}_{\varepsilon}\left(\mathrm{t}_{12}\right)=\frac{1}{\mathrm{t}_{12}^{2}}\left[\frac{\pi^{2}}{4 \varepsilon}+\frac{\pi^{2}}{4} \log \frac{\mathrm{t}_{12}^{2}}{4}+\cdots\right], \quad \widehat{D}_{ \pm, \varepsilon}\left(\mathrm{t}_{12}\right)=\frac{1}{\mathrm{t}_{12}^{2}}\left[-\frac{\pi}{4 \varepsilon}-\frac{\pi}{4} \log \frac{\mathrm{t}_{12}^{2}}{4}+\cdots\right],
$$

leading again to (4.13).

Compared to (4.8) the vanishing result in (4.13) suggests that for consistency with global symmetry (see (B.6)) the value of $g_{0}=g(w, w)$ to be used in (4.8) should be indeed

$$
g_{0}=1
$$

\subsection{One-loop correction to the three-point correlator}

Let us now compute the one-loop correction to the three-point function $\left\langle\Psi\left(t_{1}\right) \widetilde{\Psi}\left(t_{2}\right) \Phi\left(t_{3}\right)\right\rangle$ with the tree-level value given by in (2.18). ${ }^{21}$ There are two types of contributions: from the triangle diagram and its "limits", and from the "self-energy" corrections to the propagators in the tree-level diagram (2.15).

Triangle. The first is given by the following set of diagrams

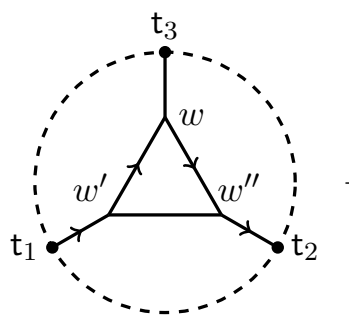

(a)

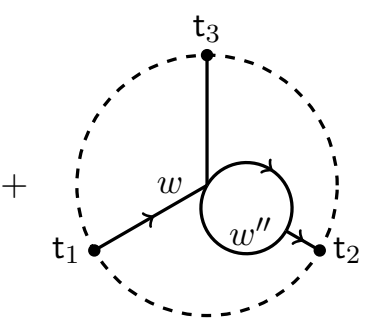

(b)

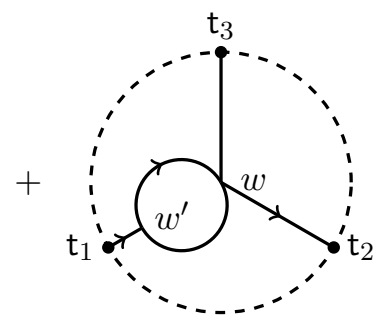

(c)

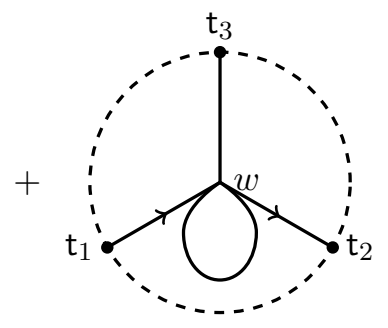

$(d)$

i.e.

$$
\left\langle\Psi\left(\mathrm{t}_{1}\right) \widetilde{\Psi}\left(\mathrm{t}_{2}\right) \Phi\left(\mathrm{t}_{3}\right)\right\rangle_{1-\text { loop }}^{\text {triangle }}=V_{a}\left(\mathrm{t}_{1}, \mathrm{t}_{2}, \mathrm{t}_{3}\right)+V_{b}\left(\mathrm{t}_{1}, \mathrm{t}_{2}, \mathrm{t}_{3}\right)+V_{c}\left(\mathrm{t}_{1}, \mathrm{t}_{2}, \mathrm{t}_{3}\right)+V_{d}\left(\mathrm{t}_{1}, \mathrm{t}_{2}, \mathrm{t}_{3}\right) .
$$

We will again separate the contributions coming from regular and $\delta$-function parts of derivatives of internal propagators in (2.12). Then the "regular" part of $V_{a}$ is given by ${ }^{22}$

$$
\begin{aligned}
V_{a}^{\mathrm{reg}} & =\left(\frac{-2 b}{\pi}\right)^{3} \int d^{2} w d^{2} w^{\prime} d^{2} w^{\prime \prime} \frac{1}{\left(\bar{w}^{\prime}-\mathrm{t}_{1}\right)^{2}} \frac{1}{\left(w^{\prime}-\bar{w}\right)^{2}} \frac{1}{\left(w-\bar{w}^{\prime \prime}\right)^{2}} \frac{1}{\left(w^{\prime \prime}-\mathrm{t}_{2}\right)^{2}} \frac{\mathrm{z}}{\left(\mathrm{t}-\mathrm{t}_{3}\right)^{2}+\mathrm{z}^{2}} g\left(w^{\prime}, w^{\prime \prime}\right) \\
& =\left(\frac{-2 b}{\pi}\right)^{3} \int d^{2} w d^{2} w^{\prime} d^{2} w^{\prime \prime} \frac{1}{\left(\bar{w}^{\prime}-\mathrm{t}_{1}\right)^{2}} \frac{\partial_{w^{\prime}} g\left(w^{\prime}, w^{\prime \prime}\right)}{w^{\prime}-\bar{w}} \frac{1}{\left(w-\bar{w}^{\prime \prime}\right)^{2}} \frac{1}{\left(w^{\prime \prime}-\mathrm{t}_{2}\right)^{2}} \frac{\mathrm{z}}{\left(\mathrm{t}-\mathrm{t}_{3}\right)^{2}+\mathrm{z}^{2}},
\end{aligned}
$$

\footnotetext{
${ }^{21}$ Similar loop corrected three-point functions in $\mathrm{AdS}_{2}$ have been considered in the Liouville theory [7], the abelian or non-abelian Toda theory [11], and the $\mathcal{N}=1$ supersymmetric Liouville theory [12]. In all those cases, the analysis has been semi-analytic because some contribution required a numerical evaluation. In the present WZW model all calculations will be fully analytical due to the simpler structure of virtual exchanges.

${ }^{22}$ In the integration by parts we may ignore the $\delta$-function from $\partial_{w^{\prime}} \frac{1}{\bar{w}^{\prime}-t_{1}}$ and its derivatives as they localize the bulk integral to the boundary.
} 
This is the triple integral over a half-plane of a rational integrand. Applying the residue theorem gives

$$
V_{a}^{\mathrm{reg}}\left(\mathrm{t}_{1}, \mathrm{t}_{2}, \mathrm{t}_{3}\right)=\left(\frac{-2 b}{\pi}\right)^{3} \frac{\pi^{3}}{4} \frac{i}{\mathrm{t}_{12} \mathrm{t}_{13} \mathrm{t}_{23}}=-\frac{2 i b^{3}}{\mathrm{t}_{12} \mathrm{t}_{13} \mathrm{t}_{23}} .
$$

The contribution $V_{a}^{\delta}$ with only one $\delta$-function from (2.12) turns out to precisely cancel the regular parts of the contributions of the two diagrams $(b)$ and $(c)$, i.e. $V_{b}^{\text {reg }}+V_{c}^{\text {reg }}$.

The contributions of the diagrams with the $\delta$-function parts of the derivatives of all of the internal $(\psi, \tilde{\psi})$ propagators reduce to that of the diagram $(d)$ (with different overall factors). Explicitly, the contribution $V_{a}^{2 \delta}$ with the two $\delta$-functions $\delta^{(2)}\left(w-w^{\prime}\right), \delta^{(2)}\left(w-w^{\prime \prime}\right)$ in the diagram $(a)$ is given by $V_{a}^{2 \delta}=-2 V_{d}$ (accounting for the symmetry factor of the $\phi$ loop) and $V_{b}^{1 \delta}=V_{c}^{1 \delta}=-V_{a}^{2 \delta}$, i.e.

$$
V^{\delta}\left(\mathrm{t}_{1}, \mathrm{t}_{2}, \mathrm{t}_{3}\right)=V_{a}^{2 \delta}+V_{b}^{1 \delta}+V_{c}^{1 \delta}+V_{d}=\frac{1}{2} V_{a}^{2 \delta}=\frac{2 i b^{3} g(w, w)}{\mathrm{t}_{12} \mathrm{t}_{23} \mathrm{t}_{13}} .
$$

Thus finally (using the notation $g_{0}$ in (4.8))

$$
\left\langle\Psi\left(\mathrm{t}_{1}\right) \widetilde{\Psi}\left(\mathrm{t}_{2}\right) \Phi\left(\mathrm{t}_{3}\right)\right\rangle_{1-\text { loop }}^{\text {triangle }}=-\frac{2 i b^{3}}{\mathrm{t}_{12} \mathrm{t}_{23} \mathrm{t}_{13}}\left(1-g_{0}\right) .
$$

Self-energy corrections. The contribution of the corresponding diagrams (here gray circles stand for sums of relevant one-loop diagrams as in (4.1) and (4.9), (4.12))

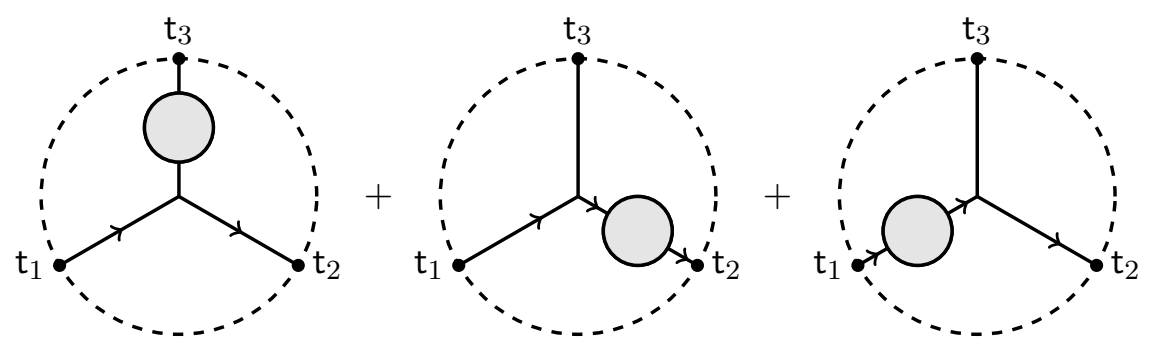

may be represented as

$$
\left\langle\Psi\left(\mathrm{t}_{1}\right) \widetilde{\Psi}\left(\mathrm{t}_{2}\right) \Phi\left(\mathrm{t}_{3}\right)\right\rangle_{1-\text { loop }}^{\text {selfengy }}=\left\langle\Psi\left(\mathrm{t}_{1}\right) \widetilde{\Psi}\left(\mathrm{t}_{2}\right) \Phi\left(\mathrm{t}_{3}\right)\right\rangle_{\text {tree }} \times\left(\frac{\langle\Phi \Phi\rangle_{1-\text { loop }}}{\langle\Phi \Phi\rangle_{\text {tree }}}+2 \frac{\langle\Psi \widetilde{\Psi}\rangle_{1 \text {-loop }}}{\langle\Psi \widetilde{\Psi}\rangle_{\text {tree }}}\right)
$$

with the full 1-loop correction to three-point function thus given by

$$
\left\langle\Psi\left(\mathrm{t}_{1}\right) \widetilde{\Psi}\left(\mathrm{t}_{2}\right) \Phi\left(\mathrm{t}_{3}\right)\right\rangle_{1-\text { loop }}=\left\langle\Psi\left(\mathrm{t}_{1}\right) \widetilde{\Psi}\left(\mathrm{t}_{2}\right) \Phi\left(\mathrm{t}_{3}\right)\right\rangle_{1 \text {-loop }}^{\text {triangle }}+\left\langle\Psi\left(\mathrm{t}_{1}\right) \widetilde{\Psi}\left(\mathrm{t}_{2}\right) \Phi\left(\mathrm{t}_{3}\right)\right\rangle_{1-\text { loop }}^{\text {self-energy }} .
$$

In view of (4.22) to (4.8), (4.13) we conclude that for the special scheme choice (4.16) under which the two-point functions do not receive one-loop corrections the same is true also for the three-point function (4.25). Then comparing to the correlators of chiral currents in $(2.37),(2.38)$ this suggests that the coefficient $\kappa$ in (2.40) does not receive quantum corrections. $^{23}$

\footnotetext{
${ }^{23} \mathrm{We}$ are assuming that the quantum theory is defined by the path integral with the WZW action (2.1), (2.3), (2.6) where the overall coefficient $k$ or $b$ in (2.6) has its classical value (an action with a shifted $k$ would correspond to a different scheme choice). It is not clear if the quantum effective action $[31,32]$ given by the WZW action with $k \rightarrow k+c_{G}$ (that reproduces correlators of currents computed in perturbation theory on a plane) is a possible starting point in computing boundary correlators of elementary fields of the WZW theory in $\mathrm{AdS}_{2}$.
} 
In appendix $\mathrm{C}$ we will further elaborate on the issue of the scheme dependence of the one-loop corrections to the boundary correlators starting with a classically equivalent action in terms of redefined fields.

\section{Boundary correlators and scattering amplitudes on $\mathrm{AdS}_{2}$}

While the scattering amplitudes for the massless WZW fields in flat space is known to vanish $[4,20]$, we have seen that the coordinate-space boundary correlators for WZW fields $\mathrm{AdS}_{2}$ are non zero. Their structure, however, is simple being dictated by the KM symmetry. One may wonder if with some natural definition of the AdS S-matrix they may actually correspond to trivial scattering in $\mathrm{AdS}_{2}$ or on half-plane. Below we will attempt to clarify this issue. ${ }^{24}$

It is useful first to recall what happened in the Liouville theory - how triviality of scattering in $\mathrm{AdS}_{2}$ emerges in that case. The flat space scattering in this theory was argued to be trivial in [21], based on previous results about the energy-momentum eigenstates in finite volume [33-36]. ${ }^{25}$ The scattering in a non-trivial Liouville vacuum or effectively in $\mathrm{AdS}_{2}$ space was discussed in [5]. ${ }^{26}$ Ref. [5] have shown that at the tree level there exists a perturbative expansion which is infrared safe and leads to trivial S-matrix. This conclusion was generalized and proved in more formal way in [22].

One may attempt to define S-matrix in AdS space by specifying suitable "in" and "out" states and computing amputated bulk correlators (as in flat space LSZ formula). In addition to the question of which asymptotic states to use (cf. [23]) a major technical problem is how to explicitly construct the Lorentzian AdS scattering amplitudes starting directly from the Euclidean coordinate-space boundary correlators.

Below we shall first outline the general relation between the AdS scattering amplitudes and the Lorentzian boundary correlators. Then we shall discuss the Euclidean $\rightarrow$ Lorentzian correlator reconstruction problem in the case of the Liouville theory relating it to the approach of [5]. Finally, we shall comment on the simplest scattering amplitude in the WZW theory in $\mathrm{AdS}_{2}$ using an analogous method.

\footnotetext{
${ }^{24}$ Starting with scattering amplitudes in AdS, one can in principle define a flat space limit of the associated S-matrix, but this requires to scale the masses/dimensions of external particles. In the massless $2 \mathrm{~d}$ case, it is unclear how to do this and whether it is possible to directly related the AdS S-matrix to the flat space one.

${ }^{25}$ To avoid infrared problems, the theory may considered on a circle, where the Liouville field $\varphi$ can be expressed in terms of a free field $\varphi^{(0)}$ by means of a quantum Bäcklund transformation. All energymomentum eigenstates on the circle can be obtained by acting on the vacuum with the modes of the stress tensor $T_{m n}^{(0)}$ of the Bäcklund field. In [21], it was argued that the dynamical properties of the infinite volume multi-particle states are equivalent to the large radius limit of the (free) $T_{m n}^{(0)}$ eigenstates. This implies that the S-matrix is trivial.

${ }^{26}$ As a normalizable translation-invariant ground state does not exist in Liouville theory in flat space, ref. [5] considered, following [8], the theory in a non-invariant domain-wall background that spontaneously breaks translation invariance and "semi-compactifies" space to a half-line. The resulting model can be identified with the Liouville theory in $\mathrm{AdS}_{2}$ geometry.
} 


\subsection{Massive scalar S-matrix on $\mathrm{AdS}_{2}$}

Let us start a scalar field theory in $\mathrm{AdS}_{2}$ with mass parameter $m^{2}=\Delta(\Delta-1)$. Let us consider a Witten diagram with one propagator connected to a bulk point $(t, \mathrm{z})$ (here $t$ is real Minkowski time, and $z \geq 0$ is the radial $\mathrm{AdS}_{2}$ Poincare coordinate). Ignoring dependence on other external points, it may be symbolically represented $\operatorname{as}^{27}$

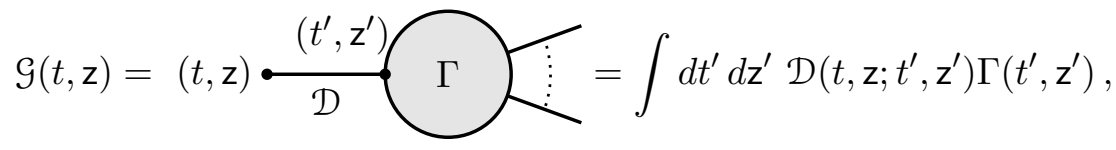

where $\mathcal{D} \equiv G_{\Delta}$ is the Lorentzian massive scalar propagator with Dirichlet boundary con$\operatorname{ditions}^{28}$

$$
\mathcal{D}\left(t, \mathbf{z} ; t^{\prime}, \mathbf{z}^{\prime}\right)=\frac{\mathfrak{C}_{\Delta}}{(2 u)^{\Delta}}{ }_{2} F_{1}\left(\Delta, \Delta, 2 \Delta,-\frac{2}{u}\right), \quad \mathcal{C}_{\Delta}=\frac{\Gamma(\Delta)}{2 \sqrt{\pi} \Gamma(\Delta+1 / 2)}, \quad u\left(x, x^{\prime}\right)=\frac{\left(\mathbf{z}-\mathbf{z}^{\prime}\right)^{2}-\left(t-t^{\prime}\right)^{2}}{2 \mathbf{z z}^{\prime}},
$$

and $\Gamma$ stands for the rest of the diagram (i.e. with one line amputated). The propagator $\mathcal{D}$ may be written as

$$
\mathcal{D}\left(t, \mathbf{z} ; t^{\prime}, \mathbf{z}^{\prime}\right)=\frac{1}{2} \int_{0}^{\infty} \frac{d \omega}{\omega} e^{-i \omega\left|t-t^{\prime}\right|} f_{\omega}(\mathbf{z}) f_{\omega}\left(\mathbf{z}^{\prime}\right)
$$

where the functions $\left\{f_{\omega}(\mathbf{z})\right\}_{\omega>0}$ are eigenmodes of the kinetic operator for a scalar field in $\mathrm{AdS}_{2}$

$$
\left(\partial_{\mathbf{z}}^{2}+\omega^{2}-\frac{m^{2}}{\mathbf{z}^{2}}\right) f_{\omega}(\mathbf{z})=0 .
$$

They form a basis in $z \in[0, \infty)$ with normalization

$$
\int_{0}^{\infty} d \omega f_{\omega}(\mathbf{z}) f_{\omega}\left(\mathbf{z}^{\prime}\right)=\delta\left(\mathbf{z}-\mathbf{z}^{\prime}\right), \quad \int_{0}^{\infty} d \mathbf{z} f_{\omega}(\mathbf{z}) f_{\omega^{\prime}}(\mathbf{z})=\delta\left(\omega-\omega^{\prime}\right) .
$$

$f_{\omega}(\mathbf{z})$ can be identified with the wave function of the asymptotic state with energy $\omega$ created by the scalar field. Its explicit form for the Dirichlet boundary condition is

$$
f_{\omega}(\mathbf{z})=a(\omega) \sqrt{\mathbf{z}} J_{\Delta-\frac{1}{2}}(\omega \mathbf{z}),
$$

where the normalization $a(\omega)$ is determined by (5.5). The corresponding scattering amplitude $\mathcal{A}\left(\omega_{1}, \ldots, \omega_{N}\right)$ may be formally defined as

$$
\mathcal{A}\left(\omega_{1}, \ldots, \omega_{N}\right)=\int\left(\prod_{i=1}^{N} d t_{i} d \mathrm{z}_{i} e^{i \omega_{i} t_{i}} f_{\omega_{i}}^{\left(\Delta_{i}\right)}\left(\mathrm{z}_{i}\right)\right) \Gamma\left(t_{1}, \mathrm{z}_{1} ; \ldots ; t_{N}, \mathrm{z}_{N}\right),
$$

where in $\Gamma$ we included the external leg labels and the subscript in $f_{\omega}^{(\Delta)}(z)$ is (5.6) indicates the corresponding value of $\Delta$ (in the case of multi-scalar scattering with different masses).

\footnotetext{
${ }^{27}$ Here $t$ is Minkowski time related to Euclidean $\mathrm{AdS}_{2}$ time $\mathrm{t}$ used above by $t=i \mathrm{t}$.

${ }^{28} \mathrm{As}$ in (2.8), this is for the standard normalization of the action, i.e. $S=\frac{1}{2} \int d^{2} x \sqrt{g}\left[(\partial \phi)^{2}+m^{2} \phi^{2}+\cdots\right]$.
} 


\subsubsection{Comments on relation to boundary correlators}

It is possible to formally "derive" a relation between (5.7) and a Fourier transform of the coordinate-space boundary correlators. Let us consider one leg in (5.1) taken to the boundary, i.e. define the boundary correlator

$$
\mathrm{A}(t)=\lim _{\mathrm{z} \rightarrow 0} \mathrm{z}^{-\Delta} \mathcal{G}(t, \mathrm{z})=t \stackrel{\left(t^{\prime}, \mathrm{z}^{\prime}\right)}{\mathcal{D}} \Gamma
$$

where the circular line on the left denotes $\mathrm{AdS}_{2}$ boundary. Substituting (5.3) into (5.1) and computing (5.8) using that $f_{\omega}(\omega z) \sim z^{\Delta}$ for $z \rightarrow 0$, we find that the Fourier transform of $\mathrm{A}(t)$ is actually the same as the scattering amplitude in (5.7). Indeed, (here $c_{\Delta}$ is a coefficient dependent only on $\Delta)^{29}$

$$
\begin{aligned}
\mathcal{A}(\omega) \equiv \int d t e^{i \omega t} \mathrm{~A}(t) & =c_{\Delta} \int d t e^{i \omega t} \int d t^{\prime} d \mathbf{z}^{\prime} \int_{0}^{\infty} \frac{d \omega^{\prime}}{\omega^{\prime}} e^{-i \omega^{\prime}\left|t-t^{\prime}\right|} a\left(\omega^{\prime}\right) \omega^{\prime \Delta} f_{\omega^{\prime}}\left(\mathbf{z}^{\prime}\right) \Gamma\left(t^{\prime}, \mathbf{z}^{\prime}\right) \\
& =c_{\Delta} \int d t^{\prime} d \mathbf{z}^{\prime} e^{i \omega t^{\prime}} \int_{0}^{\infty} \frac{d \omega^{\prime}}{\omega^{\prime}} \frac{i a\left(\omega^{\prime}\right) \omega^{\prime \Delta}}{\omega^{2}-\omega^{\prime 2}} f_{\omega^{\prime}}\left(\mathbf{z}^{\prime}\right) \Gamma\left(t^{\prime}, \mathbf{z}^{\prime}\right)
\end{aligned}
$$

Evaluating the integral over $\omega^{\prime}$ by picking (one-half of) the contribution from the pole at $\omega^{\prime}=\omega$, we obtain

$$
\mathcal{A}(\omega)=\mathcal{N}(\omega) \int d t d \mathbf{z} e^{i \omega t} f_{\omega}(\mathbf{z}) \Gamma(t, \mathbf{z}),
$$

in agreement with (5.7). The same result is found by directly considering the boundary limit of (5.1). This amounts to replacing the bulk propagator $\mathcal{D}$ by the bulk-to-boundary expression

$$
\mathrm{A}(t)=\lim _{\mathbf{z} \rightarrow 0} \mathrm{z}^{-\Delta} \mathcal{G}(t, \mathbf{z})=\mathcal{C}_{\Delta} \int d t^{\prime} d \mathbf{z}^{\prime}\left[\frac{\mathrm{z}^{\prime}}{-\left(t-t^{\prime}\right)^{2}+\mathrm{z}^{\prime 2}}\right]^{\Delta} \Gamma\left(t^{\prime}, \mathbf{z}^{\prime}\right)
$$

and taking the Fourier transform of (5.11) in Cauchy principal value sense (i.e. summing half of the two residues at $\left.t=t^{\prime} \pm \mathrm{z}^{\prime}\right) .{ }^{30}$ For $\omega>0$, it reads

$$
\mathcal{C}_{\Delta} f d t e^{i \omega t}\left[\frac{\mathbf{z}^{\prime}}{-\left(t-t^{\prime}\right)^{2}+\mathrm{z}^{\prime 2}}\right]^{\Delta}=\frac{2^{-\frac{1}{2}-\Delta} \pi}{\Gamma(\Delta+1 / 2)} \omega^{\Delta+\frac{1}{2}} \sqrt{\mathbf{z}} J_{\Delta-1 / 2}(\omega \mathbf{z}) e^{i \omega t^{\prime}}
$$

and thus implies again (5.10).

To summarize, we have shown that under a certain prescription, one can start with the $N$-leg boundary correlator for fields with dual conformal dimensions $\Delta_{1}, \ldots, \Delta_{N}$

$$
\mathrm{A}\left(t_{1}, \ldots, t_{N}\right)=\lim _{\mathrm{z}_{i} \rightarrow 0} \mathrm{z}_{1}^{-\Delta_{1}} \cdots \mathrm{z}_{N}^{-\Delta_{N}} \int\left(\prod_{i=1}^{N} d t_{i}^{\prime} d \mathrm{z}_{i}^{\prime} \mathcal{D}\left(t_{i}, \mathrm{z}_{i} ; t_{i}^{\prime}, \mathrm{z}_{i}^{\prime}\right)\right) \Gamma\left(t_{1}^{\prime}, \mathrm{z}_{1}^{\prime} ; \cdots ; t_{N}^{\prime}, \mathrm{z}_{N}^{\prime}\right)
$$

\footnotetext{
${ }^{29}$ Here and in the following, integrals $z$ are restricted to the $\mathrm{AdS}_{2}$ region, i.e. $z \geq 0$.

${ }^{30} \mathrm{At}$ this stage this is just a formal prescription. More precisely, one should shift the integration contour by adding causal $i \varepsilon$ shifts, see below.
} 
take its Fourier transform in each leg and as result find an alternative representation for the scattering amplitude $\mathcal{A}\left(\omega_{1}, \ldots, \omega_{N}\right)$ in (5.7), i.e.

$$
\mathcal{A}\left(\omega_{1}, \ldots, \omega_{N}\right)=\int\left(\prod_{i=1}^{N} d t_{i} e^{i \omega_{i} t_{i}}\right) \mathrm{A}\left(t_{1}, \ldots, t_{N}\right) .
$$

Let us note that the amputated Green's function $\Gamma$ in (5.7), as well as the boundary correlator in (5.14), are the Lorentzian ones. In general, the explicit analytical continuation of the boundary correlators from the Euclidean to the Lorentz signature should be done according to the general prescriptions based on reconstruction theorems [37] as discussed more recently in [38-40]. In particular, to compute the fully time-ordered Wightman function from the Euclidean correlators, one replaces $t_{i} \rightarrow t_{i}-i \varepsilon_{i}$ with $\varepsilon_{i}>\varepsilon_{j}$ when $t_{i}>t_{j}$ and then takes $\varepsilon_{i} \rightarrow 0 .{ }^{31}$ The Fourier transform of the resulting expression is expected to give the scattering amplitude and to match (5.7).

\subsubsection{Tree level scattering in Liouville theory on $\mathbf{A d S}_{2}$}

To illustrate the relation between (5.7) and (5.14) let us consider again the Liouville theory following [5]. The basic $1 \rightarrow 2$ particle production process $\varphi \rightarrow \varphi+\varphi$ here is particularly simple: at tree level it involves the amputated 3-point function that is just a constant. Let us begin by (5.7). The off-shell wave functions (5.6) are

$$
f_{\alpha, \omega}(\mathrm{t}, \mathrm{z})=e^{i \alpha t} \sqrt{\omega \mathbf{z}} J_{3 / 2}(\omega \mathbf{z}), \quad \omega>0 .
$$

The on-shell condition is $\alpha^{2}=\omega^{2}$, namely $\alpha= \pm \omega$. Besides, $f_{-\omega, \omega}(\mathrm{t}, \mathrm{z})=f_{-\omega,-\omega}(\mathrm{t}, \mathrm{z})$, and we can simultaneously treat both signs of $\omega$, i.e. "in" or "out" states. Up to irrelevant constants, the scattering amplitude for a 3-particle process may be written as

$$
\mathcal{A}_{3}\left(\alpha_{1}, \omega_{1} ; \alpha_{2}, \omega_{2} ; \alpha_{3}, \omega_{3}\right) \sim \delta\left(\alpha_{1}+\alpha_{2}+\alpha_{3}\right) \overline{\mathcal{A}}_{3}\left(\omega_{1}, \omega_{2}, \omega_{3}\right),
$$

where

$$
\overline{\mathcal{A}}_{3}\left(\omega_{1}, \omega_{2}, \omega_{3}\right)=\int_{0}^{\infty} \frac{d \mathrm{z}}{\mathrm{z}^{2}} f_{\omega_{1}}(z) f_{\omega_{2}}(z) f_{\omega_{3}}(z)=\sqrt{\omega_{1} \omega_{2} \omega_{3}} \int_{0}^{\infty} \frac{d \mathrm{z}}{\sqrt{\mathbf{z}}} J_{3 / 2}\left(\omega_{1} \mathrm{z}\right) J_{3 / 2}\left(\omega_{2} \mathrm{z}\right) J_{3 / 2}\left(\omega_{3} \mathrm{z}\right) .
$$

We may now use the known value of the following definite integral ${ }^{32}$

$$
\int_{0}^{\infty} \frac{d \mathbf{z}}{\mathbf{z}^{\nu-1}} J_{\nu}\left(\omega_{1} \mathbf{z}\right) J_{\nu}\left(\omega_{2} \mathbf{z}\right) J_{\nu}\left(\omega_{3} \mathbf{z}\right)=\frac{2^{\nu-1} S^{2 \nu-1}}{\sqrt{\pi}\left(\omega_{1} \omega_{2} \omega_{3}\right)^{\nu} \Gamma\left(\nu+\frac{1}{2}\right)},
$$

where

$$
S=\frac{1}{4} \sqrt{\left(\omega_{1}+\omega_{2}+\omega_{3}\right)\left(-\omega_{1}+\omega_{2}+\omega_{3}\right)\left(\omega_{1}-\omega_{2}+\omega_{3}\right)\left(\omega_{1}+\omega_{2}-\omega_{3}\right)},
$$

\footnotetext{
${ }^{31}$ As first discussed in [41], the analytical continuation can be done at the level of Mellin amplitudes, see [42-44].

${ }^{32}$ Useful integrals involving three Bessel functions are discussed in [45, 46].
} 
is the area of a triangle with sides $\omega_{1}, \omega_{2}, \omega_{3}$ (if $\omega_{1}, \omega_{2}, \omega_{3}$ do not form a triangle, the integral is zero). From (5.18) ref. [5] found the following expression for (5.17)

$$
\overline{\mathcal{A}}_{3}=\sqrt{\frac{2}{\pi}} \frac{\mathrm{S}^{2}}{\omega_{1} \omega_{2} \omega_{3}} .
$$

As the kinematically allowed 3-particle processes are associated with a degenerate triangle with vanishing area $\mathrm{S}=0$ one finds that $\mathcal{A}_{3}=0$. This calculation has been extended in [5] to the 4-particle scattering processes that were also found to vanish.

To try to recover (5.20) as a Fourier transform (5.14) of the boundary correlator we need first to analytically continue the Euclidean boundary 3-point function $\sim \frac{1}{\mathrm{t}_{12}^{2} \mathrm{t}_{13}^{2} \mathrm{t}_{23}^{2}}$ to the Lorenzian signature (to get the Lorentzian time-ordered 3-point function). Evaluating the associated Fourier transform seems far from trivial because the $d^{3} t$ integration region has to be split according to the time ordering and suitable $\pm i \varepsilon$ shifts have to be introduced. ${ }^{33}$ In principle, another approach is to look for an analytic continuation of the triple- $K$ integral representation of the Euclidean 3-point function [47]. Such analytic continuations have been recently discussed in [48].

Let us note that continuation to Lorentzian signature and time-like momenta requires an analytic continuation of expressions involving the Appel function and this is known to be related to triple- $J$ integrals for special arguments, see eq. (7.1) of [49] and also [45, 46]. This procedure is yet to be investigated in detail, but let us note that

$$
\int_{0}^{\infty} d z z^{1-\nu+\varepsilon} K_{\nu}\left(\omega_{1} z\right) K_{\nu}\left(\omega_{2} z\right) K_{\nu}\left(\omega_{3} z\right)=\frac{\pi^{2}}{2 \varepsilon} \frac{2^{\nu-1} \mathrm{~S}^{2 \nu-1}}{\sqrt{\pi}\left(\omega_{1} \omega_{2} \omega_{3}\right)^{\nu} \Gamma\left(\nu+\frac{1}{2}\right)}+\mathcal{O}(1) .
$$

This relation shows that with a simple (although ad hoc) regularization of the triple- $K$ integral, the triple- $J$ integral (relevant for the scattering amplitude) shows up as the residue at the singular pole. The fact that leading singularities of divergent triple $K$ integrals may contain physical objects has been discussed in the Euclidean context in [50]. It would be interesting to understand the relation between their analysis and relations like (5.21).

\subsection{Massless scattering case}

In view of the subtleties involved in extracting the scattering amplitudes from the Euclidean boundary correlators, here we shall consider massless scattering following the approach of [5] based on (5.7). Let us start with the simplest $1 \rightarrow 2$ process and emphasize the difference between models with derivative-independent scalar $\phi^{3}$ vertex and with $\phi(\partial \phi)^{2}$ $\sigma$-model type (classically) conformally invariant vertices.

For a massless scalar we have $\Delta=1$ or $\Delta-\frac{1}{2}=\frac{1}{2}$ in (5.6) and for a $\phi^{3}$ interaction vertex the analog of the integral in (5.17) representing the tree level $1 \rightarrow 2$ particle production

\footnotetext{
${ }^{33}$ The Fourier representation of the Wightman Lorentzian 3-point function with fixed time ordering $\left\langle\mathcal{O}\left(t_{1}\right) \mathcal{O}\left(t_{2}\right) \mathcal{O}\left(t_{3}\right)\right\rangle, t_{1}>t_{2}>t_{3}$ is discussed in [39].
} 
amplitude is

$$
\begin{aligned}
& \int_{0}^{\infty} \frac{d z}{\sqrt{z}} J_{1 / 2}\left(\omega_{1} z\right) J_{1 / 2}\left(\omega_{2} z\right) J_{1 / 2}\left(\omega_{3} z\right) \\
& =\frac{1}{2 \sqrt{2} \pi^{3 / 2} \sqrt{\omega_{1} \omega_{2} \omega_{3}}}\left[\Omega \log \left(\Omega^{2}\right)-\omega_{12} \log \left(\omega_{12}^{2}\right)-\omega_{13} \log \left(\omega_{13}^{2}\right)-\omega_{23} \log \left(\omega_{23}^{2}\right)\right],
\end{aligned}
$$

where we defined $\omega_{12}=\omega_{1}+\omega_{2}-\omega_{3}$, etc., and $\Omega=\omega_{1}+\omega_{2}+\omega_{3}$. The integral (5.22) does not vanish on-shell. For instance, if $\omega_{3} \rightarrow \omega_{1}+\omega_{2}$ it has a finite non-zero limit. ${ }^{34}$

In the $\sigma$-model case in flat space the 3 -point amplitude vanishes due to on-shell kinematics. This is less automatic in the $\mathrm{AdS}_{2}$ case. Let us consider the case of a general $\sigma$-model in the parametrization used in (3.2), (3.8) where the cubic vertex in the WZW case is $\sim f_{a b c} \partial X^{a} \bar{\partial} X^{b} X^{c}$. Because of antisymmetry of $f_{a b c}$ the vertex is effectively $\sim \frac{1}{2}\left(\partial X^{a} \bar{\partial} X^{b}-\bar{\partial} X^{a} \partial X^{b}\right) X^{c}$. Let us first consider the contribution of the first term and then antisymmetrize in momenta. We will need the wave functions

$$
\begin{aligned}
f_{ \pm \omega, \omega}(t, \mathbf{z}) & =e^{ \pm i \omega t} \sin (\mathbf{z} \omega) \\
\partial f_{ \pm \omega, \omega}(t, \mathbf{z}) & \propto\left(\partial_{t}+\partial_{\mathbf{z}}\right) e^{ \pm i \omega t} \sin (\mathbf{z} \omega)=\omega e^{ \pm i \omega(t+\mathbf{z})} \\
\bar{\partial} f_{ \pm \omega, \omega}(t, \mathbf{z}) & \propto\left(\partial_{t}-\partial_{\mathbf{z}}\right) e^{ \pm i \omega t} \sin (\mathbf{z} \omega)=-\omega e^{ \pm i \omega(t-\mathbf{z})} .
\end{aligned}
$$

Starting from (5.7), suppressing the group indices and defining $\alpha_{i}= \pm \omega_{i}$ we find (cf. (5.17), (5.22))

$$
\begin{aligned}
\mathcal{A}_{3}^{\prime} & =\int d t d z \partial f_{\alpha_{1}, \omega_{1}}(t, \mathbf{z}) \bar{\partial} f_{\alpha_{2}, \omega_{2}}(t, \mathbf{z}) f_{\alpha_{3}, \omega_{3}}(t, \mathbf{z}) \propto \int d t d \mathbf{z} \omega_{1} \omega_{2} e^{i \alpha_{1}(t+\mathrm{z})} e^{i \alpha_{2}(t-\mathrm{z})} e^{i \alpha_{3} t} \sin \left(\mathbf{z} \omega_{3}\right) \\
& \propto \delta\left(\alpha_{1}+\alpha_{2}+\alpha_{3}\right) \omega_{1} \omega_{2} \int_{0}^{\infty} d \mathbf{z}\left[e^{i\left(\alpha_{1}-\alpha_{2}+\alpha_{3}\right) \mathbf{z}}-e^{i\left(\alpha_{1}-\alpha_{2}-\alpha_{3}\right) \mathrm{z}}\right] \\
& =\delta\left(\alpha_{1}+\alpha_{2}+\alpha_{3}\right) \omega_{1} \omega_{2}\left[\pi \delta\left(\alpha_{1}-\alpha_{2}+\alpha_{3}\right)+\frac{i}{\alpha_{1}-\alpha_{2}+\alpha_{3}}-\pi \delta\left(\alpha_{1}-\alpha_{2}-\alpha_{3}\right)-\frac{i}{\alpha_{1}-\alpha_{2}-\alpha_{3}}\right] \\
& \propto \delta\left(\alpha_{1}+\alpha_{2}+\alpha_{3}\right) \omega_{1} \omega_{2} \frac{\alpha_{1}+\alpha_{2}}{\alpha_{1} \alpha_{2}}
\end{aligned}
$$

Here we used that $\int_{0}^{\infty} d z e^{i \omega z}=\pi \delta(\omega)+i \omega^{-1}$. We are still to antisymmetrize in $\omega_{1} \leftrightarrow \omega_{2}$, but since the expression in (5.24) is symmetric, the final result is thus zero. Thus the 3 -point scattering amplitude vanishes also in $\mathrm{AdS}_{2} \cdot{ }^{35}$

As for the 4-particle scattering amplitude, in the Liouville theory in $\mathrm{AdS}_{2}$ it was found to vanish in a non-trivial way, due to a cancellation of different contributions [5]. It would be interesting to see if it also vanishes in the WZW theory in $\mathrm{AdS}_{2}$. A possible reason of why this may happen is the absence of non-trivial structures in the corresponding Euclidean boundary correlators, i.e. the cancellation of logarithmic terms that happens in

\footnotetext{
${ }^{34}$ Notice that we can put an arbitrary scale $\mu$ in the logarithms in (5.22) since $\Omega-\omega_{12}-\omega_{13}-\omega_{23}=0$.

${ }^{35}$ Let us note that dealing with massless $2 \mathrm{~d}$ fields requires extra care. The wave function $f$ in $(5.23)$ is not vanishing for $z \rightarrow \infty$. Thus, integration by parts is not a priori allowed in (5.24). That means that the starting form of the action may be important as the contribution of boundary terms (produced by integrations by parts) may be non-trivial.
} 
the Liouville theory $[6,7]$ and that we also observed above for the WZW limit of a general $\sigma$-model. To establish this link it remains to derive the $\mathrm{AdS}_{2}$ scattering amplitudes from Euclidean boundary correlators in a systematic way.

\section{Concluding remarks}

In this paper we considered boundary correlators of elementary fields of $2 \mathrm{~d} \sigma$-models in $\mathrm{AdS}_{2}$. Similar problem appears in the study of correlators of operators on a Wilson line in the strong-coupling description in terms of the $\mathrm{AdS}_{5} \times S^{5}$ Nambu string action in the static gauge $[1,2]$. One motivation is to learn how to compute loop Witten diagrams in $\mathrm{AdS}_{2}$ in models with derivative interactions. We have observed, in particular, that the structure of four-point correlators simplifies (with logarithmic terms of the $1 \mathrm{~d}$ cross-ratio cancelling out) only in the WZW case when the $\sigma$-model has an extra KM symmetry. In that case the boundary correlation functions of the WZW fields are found to be the same as the correlators of the chiral WZW currents on the plane restricted to the real line.

Another possible motivation is related to the search for new integrable $2 \mathrm{~d} \sigma$-models using S-matrix based criteria as in the massive case. If one expands near a trivial $\sigma$ model vacuum in flat $2 \mathrm{~d}$ space one gets massless scattering amplitudes which, in general, suffer from IR ambiguities [4, 51]. If instead one considers the $\sigma$-model on $\mathrm{AdS}_{2}$ then its coordinate-space boundary correlators are better defined and one may try to find the analogs of the standard integrability constraints (S-matrix factorization and no particle creation) directly in terms of them. As any $2 \mathrm{~d} \sigma$-model is classically Weyl invariant, the tree-level problem in $\mathrm{AdS}_{2}$ is equivalent to the same problem on flat half-plane with particular (Dirichlet) boundary conditions. Hidden conserved charges that exist in a classically integrable $\sigma$-model on a plane should lead to constraints on the corresponding Euclidean boundary correlators and the associated S-matrix on half-plane. This should also extend to the quantum level if the $\sigma$-model is quantum scale invariant (like the WZW model).

\section{Acknowledgments}

We would like to thank D. Ponomarev, S. Giombi and R. Metsaev for useful discussions of related questions. MB was supported by the INFN grant GSS (Gauge Theories, Strings and Supergravity). HJ was supported by Swiss National Science Foundation. AAT was supported by the STFC grant ST/P000762/1.

\section{A Notation and conventions}

The $\mathrm{AdS}_{2}$ metric is

$$
d s^{2}=\frac{d \mathrm{t}^{2}+d \mathrm{z}^{2}}{\mathrm{z}^{2}}=-4 \frac{d w d \bar{w}}{(w-\bar{w})^{2}}, \quad w=\mathrm{t}+i \mathrm{z}, \quad \mathrm{z}>0
$$


and we use the conventions

$$
\begin{aligned}
\partial & \equiv \partial_{w}=\frac{1}{2}\left(\partial_{\mathrm{t}}-i \partial_{\mathrm{z}}\right), \quad \bar{\partial} \equiv \partial_{\bar{w}}=\frac{1}{2}\left(\partial_{\mathrm{t}}+i \partial_{\mathrm{z}}\right), \\
\epsilon^{\mathrm{tz}} & =-\epsilon^{\mathrm{zt}}=\mathrm{z}^{2}, \quad \epsilon^{w \bar{w}}=-\epsilon^{\bar{w} w}=-2 i \mathrm{z}^{2}, \quad \mathrm{~g}^{\mathrm{zz}}=\mathrm{g}^{\mathrm{tt}}=\mathrm{z}^{2}, \quad \mathrm{~g}^{w \bar{w}}=\mathrm{g}^{\bar{w} w}=2 \mathrm{z}^{2} .
\end{aligned}
$$

We also define the integration measure as follows

$$
d^{2} w=d \mathbf{z} d \mathbf{t}, \quad \quad \mathrm{d}^{2} w=\frac{d^{2} w}{\pi} .
$$

Our convention for the $\delta$-function is

$$
\delta^{(2)}(w)=\delta(\mathrm{t}) \delta(\mathbf{z}), \quad \int d^{2} w \delta^{(2)}(w) f(w)=f(0), \quad d^{2} w=d \mathbf{t} d \mathbf{z}, \quad w=\mathbf{t}+i \mathbf{z},
$$

so that one has

$$
\partial \frac{1}{\bar{w}}=\pi \delta^{(2)}(w), \quad \bar{\partial} \frac{1}{w}=\pi \delta^{(2)}(w) .
$$

The bulk propagator of a massless field in $\mathrm{AdS}_{2}$ with the action normalized as $S=$ $\int_{\mathrm{AdS}_{2}} \mathrm{~d}^{2} w \partial \phi \bar{\partial} \phi$ is given by

$$
g(\eta)=-\frac{1}{2} \log \eta\left(w, w^{\prime}\right),
$$

where the geodesic distance $\eta$ is defined in (2.9). The associated bulk-to-boundary propagator is

$$
g^{\partial}\left(\mathrm{t} ; w^{\prime}\right)=\lim _{\mathbf{z} \rightarrow 0} \frac{1}{\mathbf{z}} g\left(\mathrm{t}, \mathbf{z} ; \mathrm{t}^{\prime}, \mathbf{z}^{\prime}\right)=\frac{2 \mathbf{z}^{\prime}}{\left(\mathrm{t}^{\prime}-\mathrm{t}\right)^{2}+\mathrm{z}^{\prime 2}}=\frac{-i}{\mathrm{t}-w^{\prime}}+\frac{i}{\mathrm{t}-\bar{w}^{\prime}} .
$$

\section{B Global symmetry constraints in $\mathrm{SL}(2, \mathbb{R})$ WZW model}

Let us consider the consequences of the global invariance of the WZW action (2.6) under $g \rightarrow U g$ where $U$ is a $\mathrm{SL}(2, \mathbb{R})$ matrix that may be chosen as

$$
U=\left(\begin{array}{cc}
1+\gamma & \rho \\
\varepsilon & 1-\gamma
\end{array}\right),
$$

where $(\gamma, \rho, \varepsilon)$ are constant parameters. The infinitesimal transformation of the fields in $(2.3)$ reads

$$
\delta \phi=\sqrt{k}(-\gamma+\psi \varepsilon), \quad \delta \psi=2 \psi \gamma+\rho-\psi^{2} \varepsilon, \quad \delta \tilde{\psi}=k e^{-\frac{2}{\sqrt{k}} \phi} \varepsilon .
$$

In particular, taking $\gamma=\rho=0$ and rescaling $\varepsilon \rightarrow b \varepsilon$ where $b=\frac{2}{\sqrt{k}}$ gives

$$
\delta \phi=2 \psi \varepsilon, \quad \delta \psi=-b \psi^{2} \varepsilon, \quad \delta \tilde{\psi}=4 b^{-1} e^{-b \phi} \varepsilon .
$$

The action (2.6) is readily checked to be invariant under (B.3) (using integration by parts). Using the boundary asymptotics (2.7) we get from the $z \rightarrow 0$ limit of (B.3) the following transformation of the corresponding boundary fields

$$
\delta \Phi(\mathrm{t})=2 \Psi(\mathrm{t}) \varepsilon+\mathcal{O}\left(\varepsilon^{2}, \mathrm{z}\right), \quad \delta \Psi(\mathrm{t})=\mathcal{O}(\mathrm{z}), \quad \delta \widetilde{\Psi}(\mathrm{t})=4\left[b^{-1} \mathrm{z}^{-1}-\Phi(\mathrm{t})\right] \varepsilon+\mathcal{O}\left(\varepsilon^{2}, \mathrm{z}\right) .
$$


Assuming the computational scheme preserves the global SL(2, $\mathbb{R})$ symmetry, it then imposes constraints on the boundary correlators. In view of the symmetry rotating $\psi$ into $\tilde{\psi}$ one should have $\left\langle\Phi\left(\mathrm{t}_{1}\right) \widetilde{\Psi}\left(\mathrm{t}_{2}\right)\right\rangle=0$. Applying the variation (B.4) to this relation gives

$$
0=2\left\langle\Psi\left(\mathrm{t}_{1}\right) \widetilde{\Psi}\left(\mathrm{t}_{2}\right)\right\rangle+4\left\langle\Phi\left(\mathrm{t}_{1}\right) 4\left[b^{-1} \mathrm{z}^{-1}-\Phi\left(\mathrm{t}_{2}\right)\right]\right\rangle+\mathcal{O}(\varepsilon, \mathrm{z}) .
$$

The $\operatorname{SL}(2, \mathbb{R})$ symmetry implies that the tadpole $\langle\Phi\rangle$ should vanish ( $\phi$ is shifted by the parameter $\theta_{1}$ in (B.1)). ${ }^{36}$ We thus find the following relation

$$
\left\langle\Psi\left(\mathrm{t}_{1}\right) \widetilde{\Psi}\left(\mathrm{t}_{2}\right)\right\rangle=2\left\langle\Phi\left(\mathrm{t}_{1}\right) \Phi\left(\mathrm{t}_{2}\right)\right\rangle .
$$

This relation is expected to hold at the quantum level assuming the above $\mathrm{SL}(2, \mathbb{R})$ symmetry is preserved by the computational scheme. This is a necessary condition for matching the correlation functions of chiral currents on which $\mathrm{SL}(2, \mathbb{R})$ acts linearly.

\section{Alternative computation of one-loop boundary correlators in $\operatorname{SL}(2, \mathbb{R})$ WZW model}

Here we shall revisit the computation of the one-loop corrections to the two-point boundary correlators in $\mathrm{SL}(2, \mathbb{R})$ WZW model discussed in section 4.1. We shall use an alternative form of the action in terms of redefined field variables. Local field redefinitions are, in general, expected to leave the physical (boundary) correlators invariant provided they are properly defined (taking into account wave-function renormalization factors, etc.). ${ }^{37}$ Here we shall first follow a naive approach ignoring this subtlety.

Let us start with the action (2.6) and represent it in terms of the redefined fields $(\chi, \widetilde{\chi})$ defined by

$$
\psi=e^{-b \phi / 2} \chi, \quad \tilde{\psi}=e^{-b \phi / 2} \tilde{\chi} .
$$

Then up to the quartic terms (2.6) is given by ${ }^{38}$

$$
S=\int \mathrm{d}^{2} w\left[\partial \phi \bar{\partial} \phi+\partial \chi \bar{\partial} \widetilde{\chi}-\frac{b}{2}(\chi \bar{\partial} \widetilde{\chi} \partial \phi+\tilde{\chi} \partial \chi \bar{\partial} \phi)+\frac{b^{2}}{4} \chi \widetilde{\chi} \partial \phi \bar{\partial} \phi+\cdots\right] .
$$

Let us now compute the one-loop correction to the boundary two-point function for $\phi$, i.e. $\langle\Phi \Phi\rangle$. It receives contributions from several bubble diagrams (with the cubic vertices from (C.2)) and a self-contraction diagram (with the quartic vertex from (C.2)).

\footnotetext{
${ }^{36}$ Note that the one-loop contribution to $\langle\Phi\rangle$ given by the tadpole with $(\psi, \tilde{\psi})$ propagator computed with a cutoff $z>\varepsilon$ is linearly divergent$$
\mathrm{t}_{1} \frown \sim d^{2} w \frac{\mathrm{z}}{\left(\mathrm{t}-\mathrm{t}_{1}\right)^{2}+\mathrm{z}^{2}} \frac{1}{(2 i \mathrm{z})^{2}} \rightarrow-\frac{1}{4} \int_{-\infty}^{\infty} d t \int_{\varepsilon}^{\infty} d \mathrm{z} \frac{1}{\mathrm{z}\left(\mathrm{t}^{2}+\mathrm{z}^{2}\right)}=-\frac{\pi}{4 \varepsilon} .
$$

This divergence is to be subtracted in a $\mathrm{SL}(2, \mathbb{R})$ preserving scheme (see also discussion below (4.1)).

${ }^{37}$ This is easy to see at the tree level: redefinitions like $\varphi \rightarrow \varphi+\varphi^{2}+\ldots$ with $\varphi$ subject to the boundary conditions like (2.7) produce terms of higher order in $\mathbf{z} \rightarrow 0$ in the correlators.

${ }^{38}$ Note that the cubic term can be rewritten as $\chi \bar{\partial} \widetilde{\chi} \partial \phi+\widetilde{\chi} \partial \chi \bar{\partial} \phi=\chi(\bar{\partial} \widetilde{\chi} \partial \phi-\partial \widetilde{\chi} \bar{\partial} \phi)-\chi \widetilde{\chi} \bar{\partial} \partial \phi$. The first two terms here represent the standard WZ term, while the last term can be removed by a redefinition of $\phi$ under which an extra quartic term will be generated.
} 
There are two bubble diagrams where both cubic vertices are of the same type:

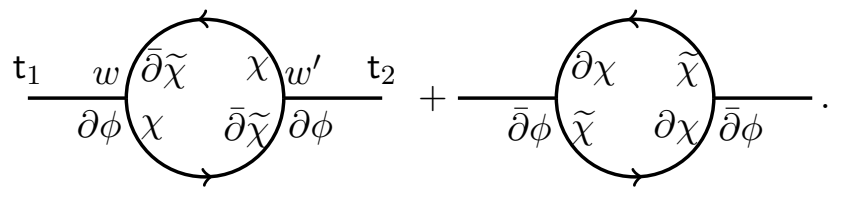

As these two diagrams are complex conjugate of each other, it is enough to focus on the contribution of the first one:

$$
\begin{aligned}
I\left(\mathrm{t}_{1}, \mathrm{t}_{2}\right) & =\int \mathrm{d}^{2} w \mathrm{~d}^{2} w^{\prime} \partial_{w} g_{\phi \phi}^{b}\left(\mathrm{t}_{1}, w\right) \partial_{w^{\prime}} g_{\phi \phi}^{b}\left(\mathrm{t}_{2}, w^{\prime}\right) \bar{\partial}_{w^{\prime}} g_{\chi \widetilde{\chi}}\left(w, w^{\prime}\right) \bar{\partial}_{w} g_{\chi \widetilde{\chi}}\left(w^{\prime}, w\right) \\
& =-\int \mathrm{d}^{2} w \mathrm{~d}^{2} w^{\prime} \frac{1}{\left(\mathrm{t}_{1}-w\right)^{2}\left(\mathrm{t}_{2}-w^{\prime}\right)^{2}} \bar{\partial}_{w^{\prime}} g_{\chi \widetilde{\chi}}\left(w, w^{\prime}\right) \bar{\partial}_{w} g_{\chi \widetilde{\chi}}\left(w^{\prime}, w\right) .
\end{aligned}
$$

Here we used the notation in (A.4), (2.10)-(2.13) (the free propagators of $\chi, \tilde{\chi}$ fields are the same as of $\psi, \tilde{\psi})$. As the integrand is a rational function one may apply the residue theorem to do the $t, t^{\prime}$ integral. It turns out that no pole survives, ${ }^{39}$ implying that the $t, t^{\prime}$ integral gives zero. Thus (C.3) gives a vanishing contribution.

The remaining bubble diagram with two different cubic vertices and the self-contraction diagram are represented by

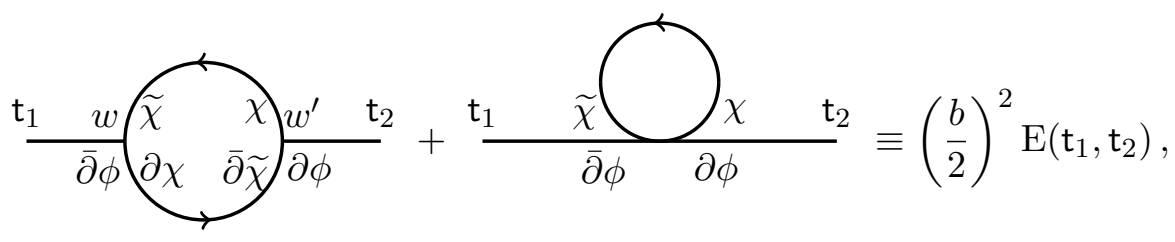

plus complex conjugate diagrams. Explicitly,

$$
\begin{aligned}
\mathrm{E}\left(\mathrm{t}_{1}, \mathrm{t}_{2}\right)= & \int \mathrm{d}^{2} w \mathrm{~d}^{2} w^{\prime} \bar{\partial}_{w} g_{\phi \phi}^{b}\left(\mathrm{t}_{1}, w\right) \partial_{w^{\prime}} g_{\phi \phi}^{b}\left(\mathrm{t}_{2}, w^{\prime}\right) \partial_{w} \bar{\partial}_{w^{\prime}} g_{\chi \widetilde{\chi}}\left(w, w^{\prime}\right) g_{\chi \widetilde{\chi}}\left(w^{\prime}, w\right) \\
& -\int \mathrm{d}^{2} w \bar{\partial}_{w} g_{\phi \phi}^{b}\left(\mathrm{t}_{1}, w\right) \partial_{w} g_{\phi \phi}^{b}\left(\mathrm{t}_{2}, w^{\prime}\right) g_{\chi \widetilde{\chi}}(w, w) \\
= & \int \mathrm{d}^{2} w \mathrm{~d}^{2} w^{\prime} \frac{1}{\left(\mathrm{t}_{1}-\bar{w}\right)^{2}\left(\mathrm{t}_{2}-w^{\prime}\right)^{2}} \frac{1}{\left(w-\bar{w}^{\prime}\right)^{2}} g_{\chi \widetilde{\chi}}\left(w^{\prime}, w\right)
\end{aligned}
$$

where the contribution of the self-contraction diagram is exactly cancelled by the part of the bubble diagram associated with the $\delta$-function piece in the derivatives of the propagator (cf. (2.12)). It is easy to see that, (4.3), (4.7) and (C.6) happen to differ by an overall factor only, although coming from different two-point functions, i.e.

$$
\mathrm{E}\left(\mathrm{t}_{1}, \mathrm{t}_{2}\right)=\frac{2}{\pi^{2}} \widehat{E}\left(\mathrm{t}_{12}\right)=\frac{1}{\mathrm{t}_{12}^{2}} .
$$

\footnotetext{
${ }^{39}$ This is true for $\mathbf{z}>\mathbf{z}^{\prime}$ and $\mathbf{z}<\mathbf{z}^{\prime}$, respectively (recall that $w=\mathbf{t}+i \mathbf{z}, w^{\prime}=\mathbf{t}^{\prime}+i \mathbf{z}^{\prime}$ ). In the case of $\mathbf{z}=\mathbf{z}^{\prime}$, one would encounter a factor of $1 /\left(t-t^{\prime}\right)^{2}$ in the integrand, which leads to a divergence when performing the $t, t^{\prime}$ integral. A more careful treatment with an explicit regularization may lead to a non-vanishing contribution, but we will not explore this here. Note that a similar subtlety happens also when $z=0$ or $\mathrm{z}^{\prime}=0$.
} 
Thus the final expression for one-loop correction is given by (taking into account the contribution of the complex conjugate to (C.5))

$$
\left\langle\Phi\left(\mathrm{t}_{1}\right) \Phi\left(\mathrm{t}_{2}\right)\right\rangle_{1-\text { loop }}=2 \times \frac{b^{2}}{4} \mathrm{E}\left(\mathrm{t}_{1}, \mathrm{t}_{2}\right)=\frac{b^{2}}{2 \mathrm{t}_{12}^{2}} .
$$

Curiously, this is different from the vanishing result in (4.13).

Assuming the symmetry relation (B.6), the result in (C.8) corresponds to

$$
\left\langle\Psi\left(\mathrm{t}_{1}\right) \tilde{\Psi}\left(\mathrm{t}_{2}\right)\right\rangle_{1 \text {-loop }}=\frac{b^{2}}{\mathrm{t}_{12}^{2}}
$$

This matches the expression in (4.8) provided one chooses $g_{0}=g(w, w)=\frac{1}{2}$ (instead of $g_{0}=1$ in $\left.(4.16)\right)$.

One may also compute the one-loop correction to the three-point function (4.25). Using (4.24) and (C.8), (C.9) we get

$$
\left\langle\Psi\left(\mathrm{t}_{1}\right) \widetilde{\Psi}\left(\mathrm{t}_{2}\right) \Phi\left(\mathrm{t}_{3}\right)\right\rangle_{1-\text { loop }}^{\text {selfenergy }}=-\frac{3 i b^{3}}{\mathrm{t}_{12} \mathrm{t}_{23} \mathrm{t}_{13}}, \quad\left\langle\Psi\left(\mathrm{t}_{1}\right) \widetilde{\Psi}\left(\mathrm{t}_{2}\right) \Phi\left(\mathrm{t}_{3}\right)\right\rangle_{1-\text { loop }}^{\text {triangle }}=\frac{2 i b^{3}}{\mathrm{t}_{12} \mathrm{t}_{23} \mathrm{t}_{13}}\left(g_{0}-1\right) .
$$

Then instead of the vanishing result in (4.25) for $g_{0}=1$ found in section 4 here we get

$$
\left\langle\Phi\left(\mathrm{t}_{1}\right) \Psi\left(\mathrm{t}_{2}\right) \tilde{\Psi}\left(\mathrm{t}_{3}\right)\right\rangle_{1-\text { loop }}=\frac{i b^{3}}{\mathrm{t}_{12} \mathrm{t}_{23} \mathrm{t}_{13}}\left(2 g_{0}-5\right)
$$

The resulting one-loop corrected expressions for the boundary correlators could be, in principle, reconciled with the corresponding correlators of the currents provided the relations between $\kappa$ and $k$ in (2.40) and between $b$ and $k$ in the action (2.4) are modified from their tree-level form.

A more consistent approach should be to define the boundary correlator with the "wave-function" renormalization factors included and that should ensure the invariance of the result under field redefinitions. Then the expressions in this appendix found starting with the redefined action (C.2) could be reconciled with the approach used in section $4 .{ }^{40}$ This remains to be clarified further.

Open Access. This article is distributed under the terms of the Creative Commons Attribution License (CC-BY 4.0), which permits any use, distribution and reproduction in any medium, provided the original author(s) and source are credited.

\footnotetext{
${ }^{40}$ One may need also to carefully take into account contributions of boundary terms from integration by parts. Note also that the use of the symmetry relation (B.6) probably requires a particular choice of the scheme, i.e. the value of the propagator at coinciding points $g(w, w)$ and its derivatives. For example, using the action (C.2) to compute explicitly the 1-loop correction of the two-point function $\langle\Psi \widetilde{\Psi}\rangle$, one would encounter the self-contraction diagram like the third diagram in (4.1). The quartic vertex in (C.2) requires us to deal with $\partial_{w} \partial_{\bar{w}} g(w, w)$ due to $\phi$ running in the loop. The regularization of such derivative term $\partial_{w} \partial_{\bar{w}} g(w, w)$ was discussed in [2].
} 


\section{References}

[1] S. Giombi, R. Roiban and A.A. Tseytlin, Half-BPS Wilson loop and $A d S_{2} / C F T_{1}$, Nucl. Phys. B 922 (2017) 499 [arXiv:1706.00756] [INSPIRE].

[2] M. Beccaria, S. Giombi and A.A. Tseytlin, Correlators on non-supersymmetric Wilson line in $\mathcal{N}=4 S Y M$ and $A d S_{2} / C F T_{1}, J H E P 05$ (2019) 122 [arXiv: 1903.04365] [INSPIRE].

[3] D. Carmi, L. Di Pietro and S. Komatsu, A Study of Quantum Field Theories in AdS at Finite Coupling, JHEP 01 (2019) 200 [arXiv:1810.04185] [INSPIRE].

[4] B. Hoare, N. Levine and A.A. Tseytlin, On the massless tree-level S-matrix in $2 d \sigma$-models, J. Phys. A 52 (2019) 144005 [arXiv:1812.02549] [InSPIRE].

[5] E. D'Hoker, D.Z. Freedman and R. Jackiw, $\mathrm{SO}(2,1)$ Invariant Quantization of the Liouville Theory, Phys. Rev. D 28 (1983) 2583 [inSPIRE].

[6] H. Ouyang, Holographic four-point functions in Toda field theories in AdS 2 , JHEP 04 (2019) 159 [arXiv: 1902.10536] [INSPIRE].

[7] M. Beccaria and A.A. Tseytlin, On boundary correlators in Liouville theory on AdS $S_{2}$, JHEP 07 (2019) 008 [arXiv: 1904.12753] [INSPIRE].

[8] E. D'Hoker and R. Jackiw, Space translation breaking and compactification in the Liouville theory, Phys. Rev. Lett. 50 (1983) 1719 [INSPIRE].

[9] A.B. Zamolodchikov and A.B. Zamolodchikov, Liouville field theory on a pseudosphere, hep-th/0101152 [INSPIRE].

[10] M. Beccaria and G. Landolfi, Toda theory in $A d S_{2}$ and $\mathcal{W} A_{n}$-algebra structure of boundary correlators, JHEP 10 (2019) 003 [arXiv:1906.06485] [INSPIRE].

[11] M. Beccaria, H. Jiang and A.A. Tseytlin, Non-abelian Toda theory on $A d S_{2}$ and $A d S_{2} / C F T_{2}^{1 / 2}$ duality, JHEP 09 (2019) 036 [arXiv: 1907.01357] [INSPIRE].

[12] M. Beccaria, H. Jiang and A.A. Tseytlin, Supersymmetric Liouville theory in AdS $S_{2}$ and AdS/CFT, JHEP 11 (2019) 051 [arXiv: 1909.10255] [INSPIRE].

[13] E. Witten, Nonabelian Bosonization in Two-Dimensions, Commun. Math. Phys. 92 (1984) 455 [INSPIRE].

[14] S.P. Novikov, The Hamiltonian formalism and a many valued analog of Morse theory, Usp. Mat. Nauk 37N5 (1982) 3 [INSPIRE].

[15] L. O'Raifeartaigh, P. Ruelle and I. Tsutsui, Quantum equivalence of constrained WZNW and Toda theories, Phys. Lett. B 258 (1991) 359 [INSPIRE].

[16] L. Feher, L. O'Raifeartaigh, P. Ruelle, I. Tsutsui and A. Wipf, On Hamiltonian reductions of the Wess-Zumino-Novikov-Witten theories, Phys. Rept. 222 (1992) 1 [InSPIRE].

[17] I.R. Klebanov and A.M. Polyakov, AdS dual of the critical $O(N)$ vector model, Phys. Lett. B 550 (2002) 213 [hep-th/0210114] [INSPIRE].

[18] J. Maldacena and A. Zhiboedov, Constraining Conformal Field Theories with A Higher Spin Symmetry, J. Phys. A 46 (2013) 214011 [arXiv:1112.1016] [INSPIRE].

[19] P. Di Francesco, P. Mathieu and D. Senechal, Conformal Field Theory, Graduate Texts in Contemporary Physics, Springer-Verlag, New York U.S.A. (1997).

[20] F.E. Figueirido, Particle creation in a conformally invariant supersymmetric model, Phys. Lett. B 227 (1989) 392 [INSPIRE]. 
[21] C.B. Thorn, The S Matrix and the Effective Potential for the Liouville Quantum Field Theory, Phys. Lett. B 128 (1983) 207 [INSPIRE].

[22] T. Yoneya, Triviality of the S Matrix in the Quantum Liouville Field Theory, Phys. Lett. B 148 (1984) 111 [INSPIRE].

[23] S.B. Giddings, The Boundary $S$ matrix and the AdS to CFT dictionary, Phys. Rev. Lett. 83 (1999) 2707 [hep-th/9903048] [INSPIRE].

[24] A. Gerasimov, A. Morozov, M. Olshanetsky, A. Marshakov and S.L. Shatashvili, Wess-Zumino-Witten model as a theory of free fields, Int. J. Mod. Phys. A 5 (1990) 2495 [INSPIRE].

[25] E. Braaten, T.L. Curtright and C.K. Zachos, Torsion and Geometrostasis in Nonlinear $\sigma$-models, Nucl. Phys. B 260 (1985) 630 [Erratum ibid. B 266 (1986) 748] [INSPIRE].

[26] A.B. Zamolodchikov, Infinite Additional Symmetries in Two-Dimensional Conformal Quantum Field Theory, Theor. Math. Phys. 65 (1985) 1205 [Teor. Mat. Fiz. 65 (1985) 347] [INSPIRE].

[27] R. Roiban, P. Sundin, A. Tseytlin and L. Wulff, The one-loop worldsheet S-matrix for the $A d S_{n} \times S^{n} \times T^{10-2 n}$ superstring, JHEP 08 (2014) 160 [arXiv:1407.7883] [INSPIRE].

[28] S. Giombi, C. Sleight and M. Taronna, Spinning AdS Loop Diagrams: Two Point Functions, JHEP 06 (2018) 030 [arXiv: 1708.08404] [INSPIRE].

[29] I. Bertan, I. Sachs and E.D. Skvortsov, Quantum $\phi^{4}$ Theory in AdS $S_{4}$ and its CFT Dual, JHEP 02 (2019) 099 [arXiv: 1810.00907] [INSPIRE].

[30] D. Meltzer, E. Perlmutter and A. Sivaramakrishnan, Unitarity Methods in AdS/CFT, JHEP 03 (2020) 061 [arXiv: 1912.09521] [inSPIRE].

[31] A.A. Tseytlin, Conformal $\sigma$-models corresponding to gauged Wess-Zumino-Witten theories, Nucl. Phys. B 411 (1994) 509 [hep-th/9302083] [INSPIRE].

[32] B. de Wit, M.T. Grisaru and P. van Nieuwenhuizen, The WZNW model at two loops, Nucl. Phys. B 408 (1993) 299 [hep-th/9307027] [INSPIRE].

[33] T.L. Curtright and C.B. Thorn, Conformally Invariant Quantization of the Liouville Theory, Phys. Rev. Lett. 48 (1982) 1309 [Erratum ibid. 48 (1982) 1768] [INSPIRE].

[34] E. Braaten, T. Curtright and C.B. Thorn, Quantum Backlund Transformation for the Liouville Theory, Phys. Lett. B 118 (1982) 115 [InSPIRE].

[35] E. Braaten, T. Curtright and C.B. Thorn, An Exact Operator Solution of the Quantum Liouville Field Theory, Annals Phys. 147 (1983) 365 [INSPIRE].

[36] J.-L. Gervais and A. Neveu, New Quantum Solution of Liouville Field Theory, Phys. Lett. B 123 (1983) 86 [INSPIRE].

[37] M. Lüscher and G. Mack, Global Conformal Invariance in Quantum Field Theory, Commun. Math. Phys. 41 (1975) 203 [INSPIRE].

[38] T. Hartman, S. Jain and S. Kundu, Causality Constraints in Conformal Field Theory, JHEP 05 (2016) 099 [arXiv: 1509.00014] [INSPIRE].

[39] T. Bautista and H. Godazgar, Lorentzian CFT 3-point functions in momentum space, JHEP 01 (2020) 142 [arXiv: 1908.04733] [INSPIRE]. 
[40] M. Gillioz, Conformal 3-point functions and the Lorentzian OPE in momentum space, arXiv: 1909.00878 [INSPIRE].

[41] G. Mack, D-independent representation of Conformal Field Theories in D dimensions via transformation to auxiliary Dual Resonance Models. Scalar amplitudes, arXiv:0907.2407 [INSPIRE].

[42] G. Mack, D-dimensional Conformal Field Theories with anomalous dimensions as Dual Resonance Models, Bulg. J. Phys. 36 (2009) 214 [arXiv:0909.1024] [INSPIRE].

[43] J. Penedones, Writing CFT correlation functions as AdS scattering amplitudes, JHEP 03 (2011) 025 [arXiv:1011.1485] [INSPIRE].

[44] A.L. Fitzpatrick, J. Kaplan, J. Penedones, S. Raju and B.C. van Rees, A Natural Language for AdS/CFT Correlators, JHEP 11 (2011) 095 [arXiv:1107.1499] [INSPIRE].

[45] A. Gervois and H. Navelet, Some Integrals Involving Three Modified Bessel Functions. 1, J. Math. Phys. 27 (1986) 682 [INSPIRE].

[46] A. Gervois and H. Navelet, Some Integrals Involving Three Modified Bessel Functions. 2, J. Math. Phys. 27 (1986) 688 [INSPIRE].

[47] A. Bzowski, P. McFadden and K. Skenderis, Implications of conformal invariance in momentum space, JHEP 03 (2014) 111 [arXiv:1304.7760] [INSPIRE].

[48] A.E. Lipstein and P. McFadden, Double copy structure and the flat space limit of conformal correlators in even dimensions, arXiv:1912.10046 [INSPIRE].

[49] W. Bailey, Some infinite integrals involving Bessel functions, Proc. Lond. Math. Soc. s2-40 (1936) 37.

[50] A. Bzowski, P. McFadden and K. Skenderis, Scalar 3-point functions in CFT: renormalisation, $\beta$-functions and anomalies, JHEP 03 (2016) 066 [arXiv:1510.08442] [INSPIRE].

[51] J.C. Donahue, S. Dubovsky, G. Hernández-Chifflet and S. Monin, From QCD Strings to WZW, JHEP 03 (2019) 120 [arXiv: 1812.07043] [INSPIRE]. 